\title{
Fast moment estimation for generalized latent Dirichlet models
}

\author{
Shiwen Zhao*1, Barbara E. Engelhardt ${ }^{\dagger 2}$, Sayan Mukherjee ${ }^{\ddagger 1}$, and David B. Dunson ${ }^{\S 1}$ \\ ${ }^{1}$ Department of Statistical Science, Duke University \\ ${ }^{2}$ Department of Computer Science, Princeton University
}

March 24, 2016

\begin{abstract}
We develop a generalized method of moments (GMM) approach for fast parameter estimation in a new class of Dirichlet latent variable models with mixed data types. Parameter estimation via GMM has been demonstrated to have computational and statistical advantages over alternative methods, such as expectation maximization, variational inference, and Markov chain Monte Carlo. The key computational advantage of our method (MELD) is that parameter estimation does not require instantiation of the latent variables. Moreover, a representational advantage of the GMM approach is that the behavior of the model is agnostic to distributional assumptions of the observations. We derive population moment conditions after marginalizing out the sample-specific Dirichlet latent variables. The moment conditions only depend on component mean parameters. We illustrate the utility of our approach on simulated data, comparing results from MELD to alternative methods, and we show the promise of our approach through the application of MELD to several data sets.
\end{abstract}

Keywords: generalized method of moments; latent Dirichlet allocation; latent variables; mixed membership model; mixed scale data; tensor factorization

*sz63@duke.edu

†bee@princeton.edu

†sayan@stat.duke.edu

$\S$ dunson@duke.edu 


\section{Introduction}

Many modern statistical applications require the analysis of large-scale, heterogeneous data types including continuous, categorical, and count variables. For example, in social science, survey data often consist of collections of different data types (e.g., height, gender, and age); in population genetics, researchers are interested in analyzing genotype (integer-valued) and heterogeneous traits (e.g., blood pressure, BMI, alcoholic drinks per day). Often data take the form of an $n \times p$ matrix $\boldsymbol{Y}=\left(\boldsymbol{y}_{1}, \ldots, \boldsymbol{y}_{n}\right)^{T}$, with $\boldsymbol{y}_{i}=\left(y_{i 1}, \ldots, y_{i p}\right)^{T}$ a $p$ dimensional vector of measurements of varying types for subject $i$, for $i=1, \ldots, n$ subjects.

This paper focuses on 1) developing a new class of generalized latent variable models for mixed data types, and 2) performing fast and robust parameter estimation using the generalized method of moments (GMM). Many models have been proposed to address the first goal (reviewed below), but often these models lack robustness both statistically and computationally. In addition, parameter estimation in these models is often both inefficient and unstable, which necessitates our second goal. Hence, there is a clear need for new classes of models and corresponding robust and efficient approaches for routine inference under these models in general applications.

For modeling mixed scale data, there are two general strategies that have been most commonly employed in the literature. The first is to assume an underlying Gaussian model, and then to characterize the dependence through a structured model of the Gaussian covariance (Muthén, 1984). Such models are routinely used in the social science literature, focusing almost entirely on the case in which data are categorical or continuous, with the categorical variables arising via thresholding of Gaussian variables. These models are related to Gaussian copula models, which have been developed for broad classes of mixed scale data also including counts (Murray et al., 2013). The second approach is to define an exponential family distribution for each of the $p$ variables, inducing dependence between variables through generalized linear models containing shared latent variables (Sammel et al. 1997; Moustaki and Knott, 2000; Dunson, 2003).

There are a number of disadvantages to the above approaches. The underlying Gaussian framework is restrictive in forcing continuous variables to be Gaussian and non-continuous variables to be categorical. The exponential family latent variable models are potentially more flexible, but in practice suffer in several respects. Most notably, there is a fundamental lack of robustness due to the model structure, which arises because of the dual role of the latent variables in controlling sample dependence and the shape of the marginal distributions. For example, if there are two count variables, $y_{i 1}$ and $y_{i 2}$, that are highly correlated, then both counts must load strongly on a similar set of latent variables, which will induce over-dispersion in the marginal distributions with the variance much larger than the mean. There is no possibility of having highly correlated counts that do not have high over-dispersion. Copula models solve this problem by imposing a restrictive Gaussian copula covariance structure. Another fundamental issue is computation, which tends to rely on expectation-maximization (EM) or Markov chain Monte Carlo (MCMC) algorithms that alternate between updating latent variables and population parameters, intrinsically leading to slow convergence, inefficiency, and instability.

One promising approach to address the above issues is to rely on a generalized method of moments (GMM) estimator, which relates to the second goal in this paper. The GMM estimator is robust to misspecification of the higher-order moments, while also leading to optimal efficiency among all estimators 
using only information on the initial moments (Hansen, 1982). Applications of GMM to structural models with latent variables have a long history, with early examples including Bentler (1983) and Anderson and Gerbing (1988), among others. More recently, Gallant et al. (2013) applied GMM to a specific class of latent variable model by defining moment conditions based on the complete data, including the latent variables. In contrast, Bollen et al. (2014) relied on a model-implied instrumental variable GMM estimator. Generally, current GMM methods focus on latent variable models that satisfy restrictive assumptions or require the instantiation of latent variables in a computationally intensive estimation algorithm.

The focus of our work is on a broad new class of latent variable models for mixed scale data. This eliminates some of the problems of current exponential family latent variable models, while also enabling derivation of an efficient GMM implementation that marginalizes out latent variables. With the first goal in mind, we focus on generalized mixed membership models, which incorporate Dirichlet latent variables. Mixed membership models have a rich literature with applications ranging from inference of ancestral populations in genomic data (Pritchard et al. 2000a b) to topic modeling of documents (Blei et al. 2003). Using Dirichlet latent variables to define cluster membership allows samples to partially belong to each of $k$ latent components. Our model class includes latent Dirichlet allocation (Blei et al. 2003) and simplex factor models (Bhattacharya and Dunson, 2012) as special cases; however, we go beyond current approaches by allowing mixed scale data.

For the second goal, we develop an efficient moment tensor approach for parameter estimation in mixed membership models with the Dirichlet latent variables marginalized out. This objective is related to recent moment tensor methods developed for latent variable models including mixtures of Gaussians, hidden Markov models, mixed membership models, and stochastic block models Arora et al., 2012, Anandkumar et al., 2012a b, Hsu and Kakade, 2013, Anandkumar et al., 2014a b). This recent work finds moment tensors of these latent variable models that have a symmetric PARAFAC (parallel factors) tensor decomposition (Kiers, 2000). Parameter estimation using a moment tensor approach is performed as follows: 1) third order empirical moment tensors are transformed to an orthogonal decomposable form; 2) orthogonal decompositions are performed using tensor power methods or singular value decompositions; 3) parameter estimates are recovered from the decomposition of the moment tensors. The moment tensor approach offers substantial computational advantages over other approximation methods, as illustrated in various applications (Tung and Smola, 2014, Anandkumar et al. 2014a; Colombo and Vlassis, 2015).

In this work, we develop a moment tensor approach for generalized mixed membership models. Here, parameter estimates are performed in the GMM framework by minimizing quadratic forms of the moment functions, and asymptotic efficiencies are analyzed. Our approach, Moment Estimation for Latent Dirichlet models (MELD), differs from previous approaches in that the moment functions are defined for second and third order moments for variables with different generative distributions. This goes beyond previous moment tensor approaches that construct moment tensors for homogeneous data distributions.

In Section 2, we characterize the class of generalized Dirichlet latent variable models in MELD. In Section 3, we briefly review GMM and introduce the estimation procedure used in MELD. Asymptotic properties of the GMM estimator are also discussed. In Section 4, we evaluate the performance in a simulation study. We apply our method to two examples in Section 5 and conclude with a discussion in Section 6 . 


\section{Generalized Dirichlet latent variable models}

In this section, we specify a generalized Dirichlet latent variable model. Let $\boldsymbol{y}_{i}=\left(y_{i 1}, \ldots, y_{i p}\right)^{T}$ denote a vector of $p$ measurements having heterogeneous data types (e.g., continuous, categorical, and counts) over subjects $i=1, \ldots, n$. We are interested in flexible models for joint distributions of the data. A simple way to achieve this is to assume the elements of $\boldsymbol{y}_{i}$ are conditionally independent given a component index $s_{i} \in\{1, \ldots, k\}$, with the densities for each variable in each component having a parametric form. This is inflexible due to the assumption that each subject $i$ belongs to exactly one component. We instead allow subjects to partially belong to each of the $k$ components. We assume that the elements of $\boldsymbol{y}_{i}$ are conditionally independent given a latent Dirichlet vector $\boldsymbol{x}_{i}=\left(x_{i 1}, \ldots, x_{i k}\right)^{T} \in \Delta^{k-1}$, with $\Delta^{k-1}$ denoting the $(k-1)$ probability simplex. In particular, we let

$$
y_{i j} \sim \sum_{h=1}^{k} x_{i h} g_{j}\left(\phi_{j h}\right),
$$

where $g_{j}\left(\phi_{j h}\right)$ is the density of the $j$ th variable specific to component $h$. The elements of $\boldsymbol{x}_{i}$ are interpretable as probability weights for subject $i$ on each of $k$ components; in this setting, pure subjects have weight vectors with all zeros except for a single one.

The corresponding density $g_{j}\left(\phi_{j h}\right)$ is absolutely continuous with respect to a dominating measure $(\Omega, \mathcal{H}, \mu)$; in general, it can take any parametric form, and may be constrained to belong to the exponential family. Our model in Equation (1) generalizes the traditional mixed membership model to allow mixed data types. The parameters $\boldsymbol{\Phi}=\left\{\boldsymbol{\phi}_{j h}\right\}_{1 \leq j \leq p, 1 \leq h \leq k}$ are mixture component parameters shared by all subjects. A full likelihood specification is completed by choosing a population distribution for the latent variable vector, $\boldsymbol{x}_{i} \sim P$, with $P$ a distribution on the simplex $\Delta^{k-1}$. In this work, we put the Dirichlet distribution on this latent variable vector $\boldsymbol{x}_{i} \sim \operatorname{Dir}(\boldsymbol{\alpha})$ with parameter $\boldsymbol{\alpha}=\left(\alpha_{1}, \ldots, \alpha_{k}\right)^{T}$.

Let $\boldsymbol{m}_{i}=\left(m_{i 1}, \ldots m_{i p}\right)^{T}$ denote a membership vector for subject $i$, where $m_{i j} \in\{1, \ldots, k\}$ indicates the component that feature $j$ in subject $i$ is generated from. We specify the following generative model

$$
y_{i j}\left|m_{i j}=h \sim g_{j}\left(\phi_{j h}\right), \quad m_{i j}\right| \boldsymbol{x}_{i} \sim \operatorname{Multi}\left(\boldsymbol{x}_{i}\right), \quad \boldsymbol{x}_{i} \sim \operatorname{Dir}(\boldsymbol{\alpha}) .
$$

When $\boldsymbol{y}_{i}$ is a multivariate categorical vector and $g_{j}$ represents a multinomial distribution, Bhattacharya and Dunson (2012) proposed a simplex factor model. Their model reduces to latent Dirichlet allocation (Blei et al. 2003 ) in the special case in which $y_{i j}$ is the number of occurrences of word $j$ in document $i$, and $g_{j}\left(\phi_{j h}\right)$ is a Poisson distribution, with scalar $\phi_{j h}$ indicating the rate of occurrence of word $j$ in topic $h$. We also assume $\boldsymbol{x}_{i} \sim \operatorname{Dir}(\boldsymbol{\alpha})$, but allow a general form for the component specific densities. This leads to the following likelihood after marginalizing out the latent variables:

$$
p(\boldsymbol{Y} \mid \boldsymbol{\alpha}, \mathbf{\Phi})=\prod_{i=1}^{n}\left[\int \prod_{j=1}^{p}\left(\sum_{h=1}^{k} x_{i h} g_{j}\left(y_{i j} \mid \boldsymbol{\phi}_{j h}\right)\right) \mathrm{d} P\left(\boldsymbol{x}_{i}\right)\right] .
$$

Categorical data: Let $y_{i j} \in\left\{1, \ldots, d_{j}\right\}$, for $j=1, \ldots, p$, so that we have multivariate categorical data that can be organized as a $p$-way contingency table. Let $c=\left(c_{1}, \ldots, c_{p}\right)^{T}$ with $c_{j} \in\left\{1, \ldots, d_{j}\right\}$ and $\pi_{\boldsymbol{c}}=\operatorname{Pr}\left(\boldsymbol{y}_{i}=\boldsymbol{c}\right)=\operatorname{Pr}\left(y_{i 1}=c_{1}, \ldots, y_{i p}=c_{p}\right)$. Then $\boldsymbol{\pi}=\left\{\pi_{\boldsymbol{c}}\right\}$ is a probability tensor (Dunson and Xing, $2009)$ satisfying $\pi_{c} \geq 0$ and $\sum_{c} \pi_{c}=1$. In this case, $g_{j}\left(\phi_{j h}\right) \stackrel{d}{=} \operatorname{Multi}\left(\phi_{j h}\right)$ is a multinomial distribution, with $\phi_{j h}=\left(\phi_{j h 1}, \ldots, \phi_{j h d_{j}}\right)^{T} \in \Delta^{d_{j}-1}$ a probability vector. As shown by Bhattacharya and Dunson 
(2012), marginalizing out the mixture proportion $\boldsymbol{x}_{i}$ leads to a Tucker decomposition of $\boldsymbol{\pi}$

$$
\pi_{\boldsymbol{c}}=\sum_{h_{1}=1}^{k} \ldots \sum_{h_{p}=1}^{k} g_{h_{1}, \ldots, h_{p}} \prod_{j=1}^{p} \phi_{j h_{j} c_{j}} .
$$

Mixed data types: When $\boldsymbol{y}_{i}$ includes variables of mixed data types, $g_{j}\left(\boldsymbol{\phi}_{j h}\right)$ belongs to the exponential family of distributions with mean parameter $\phi_{j h}$. We are interested in estimating mean parameters $\boldsymbol{\Phi}=\left(\boldsymbol{\Phi}_{1} ; \ldots ; \boldsymbol{\Phi}_{p}\right)$ with $\boldsymbol{\Phi}_{j}=\left(\phi_{j 1}, \ldots, \phi_{j k}\right)$. For categorical variables $y_{i j}, \phi_{j h}$ is a probability vector indicating the distribution of the categories in component $h$; for non-categorical variables $y_{i j}, \phi_{j h}$ is a scalar term indicating the mean parameter of that feature for component $h$.

\section{Generalized methods of moments estimation}

In this section, we provide a procedure for parameter estimation in generalized Dirichlet latent variable models based on a GMM framework. We first state the moment functions and then propose a two-stage procedure for parameter estimation.

\subsection{Moment functions used in MELD}

The idea behind method of moments (MM) algorithms is to derive a list of moment equations that have expected value zero at the true parameter values. Given observations $\left\{\boldsymbol{y}_{i}\right\}_{1 \leq i \leq n}$, MM algorithms specify $\ell$ moment functions and define a moment vector $f\left(\boldsymbol{y}_{i}, \boldsymbol{\theta}\right)=\left(f_{1}\left(\boldsymbol{y}_{i}, \boldsymbol{\theta}\right), \ldots, f_{\ell}\left(\boldsymbol{y}_{i}, \boldsymbol{\theta}\right)\right)^{T}$ such that $\mathbb{E}\left[f\left(\boldsymbol{y}_{i}, \boldsymbol{\theta}\right)\right]=\mathbf{0}$ at the true parameter $\boldsymbol{\theta}=\boldsymbol{\theta}_{0} \in \boldsymbol{\Theta} \subseteq \mathbb{R}^{p}$. The moment vector is then used to specify an estimator by solving the following $\ell$ sample equations with $p$ unknowns:

$$
\widehat{\boldsymbol{\theta}} \text { such that } \mathbf{0}=\left[f_{n}(\boldsymbol{\theta}) \equiv \frac{1}{n} \sum_{i=1}^{n} f\left(\boldsymbol{y}_{i}, \boldsymbol{\theta}\right)\right] \text { at } \boldsymbol{\theta}=\widehat{\boldsymbol{\theta}} \text {, }
$$

where $f_{n}(\boldsymbol{\theta})$ is the sample estimate of $\mathbb{E}\left[f\left(\boldsymbol{y}_{i}, \boldsymbol{\theta}\right)\right]$. When $f\left(\boldsymbol{y}_{i}, \boldsymbol{\theta}\right)$ is linear in $\boldsymbol{\theta}$ (e.g., linear regression with instrumental variables) with independent moment functions and $\ell=p$, then $\boldsymbol{\theta}$ can be uniquely determined. When $\ell>p$, the system in Equation (3) might be over-determined, in which case standard MM cannot be applied.

Generalized method of moments (GMM) minimizes the following quadratic form rather than solving a linear system:

$$
\widehat{\boldsymbol{\theta}} \equiv \arg \min _{\boldsymbol{\theta}}\left[Q_{n}\left(\boldsymbol{\theta} ; \boldsymbol{A}_{n}\right)=f_{n}(\boldsymbol{\theta})^{T} \boldsymbol{A}_{n} f_{n}(\boldsymbol{\theta})\right]
$$

with $\boldsymbol{A}_{n}$ the asymptotically optimal positive semidefinite matrix:

$$
\boldsymbol{A}_{n}^{-1}=\boldsymbol{S}_{n}=\operatorname{Var}\left[n^{1 / 2} f_{n}(\boldsymbol{\theta})\right]
$$

In the over-determined setting, minimizing the quadratic form is still well defined. Hansen's two stage procedure (Hansen, 1982) obtains an initial estimate of $\widehat{\boldsymbol{\theta}}$ using a suboptimal weight matrix, such as the identity. This initial estimate is used to calculate $\boldsymbol{A}_{n}$, which is then plugged into Equation (4) to obtain the final estimate (Hall, 2005) which is consistent and asymptotically normal (Hansen, 1982).

Applying GMM to all of the parameters in the generalized Dirichlet latent variable model is not feasible due to the dimensionality of the parameter space. Higher-order moment functions are complex and involve 
large numbers of unknowns. We will make use of a tensor decomposition and define moment functions that only depend on lower order moments. This extends recent moment tensor approaches to our proposed broad class of latent variable models (Arora et al., 2012, Anandkumar et al., 2012a b; Hsu and Kakade, 2013 Anandkumar et al. 2014a b). One key innovation in our procedure is that we allow for lower order interaction moments across variables, where each variable can have a different distribution. The moment functions will have rank one decompositions of the component mean parameters over the heterogeneous variables. These heterogeneous low-order moment functions lead to fast and robust parameter estimation in MELD, as we show in the simulation and application sections.

We now define the moment functions. Recall that $y_{i j}$ is the $j$ th variable for subject $i$, which may include various data types, including continuous, categorical, or count data. Each categorical variable $y_{i j}$ is encoded as a vector $\boldsymbol{b}_{i j}$ of length $d_{j}$ containing all zeros except for a one in position $y_{i j}$. If $y_{i j}$ is a non-categorical variable, then $\boldsymbol{b}_{i j}=y_{i j}$ is a scalar value. In the following, we assume that there are $k$ latent components and that the latent variable $\boldsymbol{x}_{i}$ follows a Dirichlet distribution with parameters $\boldsymbol{\alpha}=\left(\alpha_{1}, \ldots, \alpha_{k}\right)^{T}$ and $\alpha_{0}=\sum_{h=1}^{k} \alpha_{h}$. Given an observation $\boldsymbol{b}_{i j}$ and hyperparameter $\boldsymbol{\alpha}$, we define moment functions based on second and third moments:

$$
\begin{aligned}
& \boldsymbol{F}_{j t}^{(2)}\left(\boldsymbol{y}_{i}, \boldsymbol{\Phi}\right)=\boldsymbol{b}_{i j} \circ \boldsymbol{b}_{i t}-\frac{\alpha_{0}}{\alpha_{0}+1} \boldsymbol{\mu}_{j} \circ \boldsymbol{\mu}_{t}-\boldsymbol{\Phi}_{j} \boldsymbol{\Lambda}^{(2)} \boldsymbol{\Phi}_{t}^{T}, \quad 1 \leq j, t \leq p, j \neq t \\
& \boldsymbol{F}_{j s t}^{(3)}\left(\boldsymbol{y}_{i}, \boldsymbol{\Phi}\right)=\boldsymbol{b}_{i j} \circ \boldsymbol{b}_{i s} \circ \boldsymbol{b}_{i t} \\
& \quad-\frac{\alpha_{0}}{\alpha_{0}+2}\left(\boldsymbol{b}_{i j} \circ \boldsymbol{b}_{i s} \circ \boldsymbol{\mu}_{t}+\boldsymbol{\mu}_{j} \circ \boldsymbol{b}_{i s} \circ \boldsymbol{b}_{i t}+\boldsymbol{b}_{i j} \circ \boldsymbol{\mu}_{s} \circ \boldsymbol{b}_{i t}\right) \\
& \quad+\frac{2 \alpha_{0}^{2}}{\left(\alpha_{0}+1\right)\left(\alpha_{0}+2\right)} \boldsymbol{\mu}_{j} \circ \boldsymbol{\mu}_{s} \circ \boldsymbol{\mu}_{t}-\Lambda^{(3)} \times_{1} \boldsymbol{\Phi}_{j} \times_{2} \boldsymbol{\Phi}_{s} \times_{3} \boldsymbol{\Phi}_{t}, \\
& \quad 1 \leq j, s, t \leq p, j \neq s \neq t .
\end{aligned}
$$

We use $\circ$ to denote an outer product, and $\times_{s}$ indicates multiplication of a tensor with a matrix for mode $s$. The means $\boldsymbol{\mu}_{j}$ take the values $\boldsymbol{\mu}_{j}=\mathbb{E}\left(\boldsymbol{b}_{i j} \mid \boldsymbol{\Phi}\right)=\frac{1}{\alpha_{0}} \boldsymbol{\Phi}_{j} \boldsymbol{\alpha}$ for $j=1, \ldots, p$. The second moment function $\boldsymbol{F}_{j t}^{(2)}\left(\boldsymbol{y}_{i}, \boldsymbol{\Phi}\right)$ is a $d_{j} \times d_{t}$ matrix, and the third moment function $\boldsymbol{F}_{j s t}^{(3)}\left(\boldsymbol{y}_{i}, \boldsymbol{\Phi}\right)$ is a $d_{j} \times d_{s} \times d_{t}$ tensor. We set $d_{j}=1$ when variable $j$ is non-categorical. The matrix $\boldsymbol{\Lambda}^{(2)}$ is a $k \times k$ matrix with $\boldsymbol{\alpha} /\left[\alpha_{0}\left(\alpha_{0}+1\right)\right]$ along the diagonals and zero for all other entries. $\boldsymbol{\Lambda}^{(3)}$ is a three-way tensor with the diagonal entry $\lambda_{h}^{(3)}=2 \alpha_{h} /\left[\alpha_{0}\left(\alpha_{0}+1\right)\left(\alpha_{0}+2\right)\right]$ for $h=1, \ldots, k$ and all the other entries are zero.

The following theorem states that, at the true parameter value, the expectations of the moment functions are zero. The proof can be found in Appendix A

Theorem 1 (Moment conditions in MELD). The expectations of the second moment matrix $\boldsymbol{F}_{j t}^{(2)}\left(\boldsymbol{y}_{i}, \boldsymbol{\Phi}\right)$ and third moment tensor $\boldsymbol{F}_{j s t}^{(3)}\left(\boldsymbol{y}_{i}, \boldsymbol{\Phi}\right)$ defined in Equations (5) and (6) are zero at true model parameter values $\boldsymbol{\Phi}_{0}$

$$
\mathbb{E}\left[\boldsymbol{F}_{j t}^{(2)}\left(\boldsymbol{y}_{i}, \boldsymbol{\Phi}_{0}\right)\right]=\mathbf{0}, \quad \mathbb{E}\left[\boldsymbol{F}_{j s t}^{(3)}\left(\boldsymbol{y}_{i}, \boldsymbol{\Phi}_{0}\right)\right]=\mathbf{0}
$$

with the expectations taken with respect to $\boldsymbol{y}_{i}$.

\subsection{Two stage optimal estimation}

We will state two versions of Hansen's two stage optimal GMM estimation procedure for parameter inference in MELD, with the first version using the second moment matrix in (5) and second version using both the second moment matrix in (5) and the third moment tensor in (6). To fix notation, we re-state Hansen's two stage GMM procedure as: 
(1) estimate $\widehat{\boldsymbol{\theta}}=\arg \min _{\boldsymbol{\theta}}\left[Q_{n}(\boldsymbol{\theta} ; \boldsymbol{I})=f_{n}(\boldsymbol{\theta})^{T} f_{n}(\boldsymbol{\theta})\right]$;

(2) given $\widehat{\boldsymbol{\theta}}$ calculate $\boldsymbol{S}_{n}$ and set $\boldsymbol{A}_{n}=\boldsymbol{S}_{n}^{-1}$;

(3) compute $\widehat{\boldsymbol{\theta}}=\arg \min _{\boldsymbol{\theta}}\left[Q_{n}\left(\boldsymbol{\theta} ; \boldsymbol{A}_{n}\right)=f_{n}(\boldsymbol{\theta})^{T} \boldsymbol{A}_{n} f_{n}(\boldsymbol{\theta})\right]$ as the final parameter estimate.

We now define two moment vectors used in MELD. We define the first version of moment vector $\boldsymbol{f}^{(2)}\left(\boldsymbol{y}_{i}, \boldsymbol{\Phi}\right)$ by stacking second moment matrices in Equation (5) as follows:

$$
\begin{aligned}
\boldsymbol{f}^{(2)}\left(\boldsymbol{y}_{i}, \boldsymbol{\Phi}\right)= & \left(\operatorname{vec}\left[\boldsymbol{F}_{12}^{(2)}\left(\boldsymbol{y}_{i}, \boldsymbol{\Phi}\right)\right]^{T}, \ldots, \operatorname{vec}\left[\boldsymbol{F}_{1 p}^{(2)}\left(\boldsymbol{y}_{i}, \boldsymbol{\Phi}\right)\right]^{T},\right. \\
& \left.\operatorname{vec}\left[\boldsymbol{F}_{23}^{(2)}\left(\boldsymbol{y}_{i}, \boldsymbol{\Phi}\right)\right]^{T}, \ldots, \operatorname{vec}\left[\boldsymbol{F}_{2 p}^{(2)}\left(\boldsymbol{y}_{i}, \boldsymbol{\Phi}\right)\right]^{T}, \ldots, \operatorname{vec}\left[\boldsymbol{F}_{p-1, p}^{(2)}\left(\boldsymbol{y}_{i}, \boldsymbol{\Phi}\right)\right]^{T}\right)^{T} .
\end{aligned}
$$

As the moment matrix is symmetric in the sense that $\boldsymbol{F}_{j t}^{(2)}\left(\boldsymbol{y}_{i}, \boldsymbol{\Phi}\right)=\left[\boldsymbol{F}_{t j}^{(2)}\left(\boldsymbol{y}_{i}, \boldsymbol{\Phi}\right)\right]^{T}$, we only consider the case where $j<t$. Assuming $d$ levels for each element of $\boldsymbol{y}_{i}$ results in a moment vector of dimension $p(p-1) d^{2} / 2$. The second version of moment vector $\boldsymbol{f}^{(3)}\left(\boldsymbol{y}_{i}, \boldsymbol{\Phi}\right)$ is defined by stacking the second moment matrices and third moment tensors in Equations (5) and (6)

$$
\begin{aligned}
\boldsymbol{f}^{(3)}\left(\boldsymbol{y}_{i}, \boldsymbol{\Phi}\right)= & \left(\operatorname{vec}\left[\boldsymbol{F}_{12}^{(2)}\left(\boldsymbol{y}_{i}, \boldsymbol{\Phi}\right)\right]^{T}, \ldots, \operatorname{vec}\left[\boldsymbol{F}_{p-1, p}^{(2)}\left(\boldsymbol{y}_{i}, \boldsymbol{\Phi}\right)\right]^{T},\right. \\
& \operatorname{vec}\left[\boldsymbol{F}_{123}^{(3)}\left(\boldsymbol{y}_{i}, \boldsymbol{\Phi}\right)\right]^{T}, \ldots, \operatorname{vec}\left[\boldsymbol{F}_{12 p}^{(3)}\left(\boldsymbol{y}_{i}, \boldsymbol{\Phi}\right)\right]^{T}, \\
& \left.\operatorname{vec}\left[\boldsymbol{F}_{134}^{(3)}\left(\boldsymbol{y}_{i}, \boldsymbol{\Phi}\right)\right]^{T}, \ldots, \operatorname{vec}\left[\boldsymbol{F}_{13 p}^{(3)}\left(\boldsymbol{y}_{i}, \boldsymbol{\Phi}\right)\right]^{T} \ldots, \operatorname{vec}\left[\boldsymbol{F}_{p-2, p-1, p}^{(3)}\left(\boldsymbol{y}_{i}, \boldsymbol{\Phi}\right)\right]^{T}\right)^{T} .
\end{aligned}
$$

The moment matrices $\boldsymbol{F}_{j t}^{(2)}\left(\boldsymbol{y}_{i}, \boldsymbol{\Phi}\right)$ follow the same order as in $\boldsymbol{f}^{(2)}\left(\boldsymbol{y}_{i}, \boldsymbol{\Phi}\right)$. The third moment tensors $\boldsymbol{F}_{j s t}^{(3)}\left(\boldsymbol{y}_{i}, \boldsymbol{\Phi}\right)$ are ordered such that the index on the right runs faster than the index on the left in the subscript. The third moment tensor is symmetric with respect to any permutations of its indices, so we include only the moment tensors with $j<s<t$. The dimension of this moment vector is $p(p-1) d^{2} / 2+$ $\left[p^{3}-3 p(p-1)-p\right] d^{3} / 6$ with the assumption of $d$ levels for each element of $\boldsymbol{y}_{i}$.

Based on the two moment vector versions, we define the following two quadratic functions used for our GMM estimation:

$$
\begin{aligned}
& Q_{n}^{(2)}\left(\boldsymbol{\Phi} ; \boldsymbol{A}_{n}^{(2)}\right)=\boldsymbol{f}_{n}^{(2)}(\boldsymbol{\Phi})^{T} \boldsymbol{A}_{n}^{(2)} \boldsymbol{f}_{n}^{(2)}(\boldsymbol{\Phi}), \\
& Q_{n}^{(3)}\left(\boldsymbol{\Phi} ; \boldsymbol{A}_{n}^{(3)}\right)=\boldsymbol{f}_{n}^{(3)}(\boldsymbol{\Phi})^{T} \boldsymbol{A}_{n}^{(3)} \boldsymbol{f}_{n}^{(3)}(\boldsymbol{\Phi}),
\end{aligned}
$$

where $\boldsymbol{f}_{n}^{(2)}(\boldsymbol{\Phi})$ and $\boldsymbol{f}_{n}^{(3)}(\boldsymbol{\Phi})$ are sample estimates of the expectations of the moment vectors

$$
\boldsymbol{f}_{n}^{(2)}(\boldsymbol{\Phi})=\frac{1}{n} \sum_{i=1}^{n} \boldsymbol{f}^{(2)}\left(\boldsymbol{y}_{i}, \boldsymbol{\Phi}\right), \quad \boldsymbol{f}_{n}^{(3)}(\boldsymbol{\Phi})=\frac{1}{n} \sum_{i=1}^{n} \boldsymbol{f}^{(3)}\left(\boldsymbol{y}_{i}, \boldsymbol{\Phi}\right),
$$

and $\boldsymbol{A}_{n}^{(2)}$ and $\boldsymbol{A}_{n}^{(3)}$ are two positive semidefinite matrices. We emphasize that when calculating $\boldsymbol{f}_{n}^{(2)}(\boldsymbol{\Phi})$, the $\boldsymbol{\mu}_{j}$ and $\boldsymbol{\mu}_{t}$ in $\boldsymbol{f}^{(2)}\left(\boldsymbol{y}_{i}, \boldsymbol{\Phi}\right)$ are replaced by their sample estimates $\widehat{\boldsymbol{\mu}}_{j}=\frac{1}{n} \sum_{i=1}^{n} \boldsymbol{b}_{i j}$ and $\widehat{\boldsymbol{\mu}}_{t}=\frac{1}{n} \sum_{i=1}^{n} \boldsymbol{b}_{i t}$, instead of their parametric counterparts $\frac{1}{\alpha_{0}} \boldsymbol{\Phi}_{j} \boldsymbol{\alpha}$ and $\frac{1}{\alpha_{0}} \boldsymbol{\Phi}_{t} \boldsymbol{\alpha}$. In doing this the second moment matrix $\boldsymbol{F}_{j t}^{(2)}\left(\boldsymbol{y}_{i}, \boldsymbol{\Phi}\right)$ becomes a function of $\boldsymbol{\Phi}$ only through $\boldsymbol{\Phi}_{j} \Lambda^{(2)} \boldsymbol{\Phi}_{t}^{T}$, which is a rank one summation of $\phi_{j h}$ and $\phi_{t h}$. This method facilitates development of a fast coordinate descent algorithm for parameter estimation. Similar methods are used for calculating $\boldsymbol{f}_{n}^{(3)}(\boldsymbol{\Phi})$.

In the first stage, we set $\boldsymbol{A}_{n}^{(\cdot)}$ to an identity weight matrix. Then the quadratic functions in Equations (9) and (10) can be re-written as follows

$$
Q_{n}^{(2)}(\boldsymbol{\Phi}, \boldsymbol{I})=\sum_{j=1}^{p-1} \sum_{t=j+1}^{p}\left\|\boldsymbol{F}_{n, j t}^{(2)}(\boldsymbol{\Phi})\right\|_{F}^{2},
$$




$$
Q_{n}^{(3)}(\boldsymbol{\Phi}, \boldsymbol{I})=\sum_{j=1}^{p-1} \sum_{t=j+1}^{p}\left\|\boldsymbol{F}_{n, j t}^{(2)}(\boldsymbol{\Phi})\right\|_{F}^{2}+\sum_{j=1}^{p-2} \sum_{s=j+1}^{p-1} \sum_{t=s+1}^{p}\left\|\boldsymbol{F}_{n, j s t}^{(3)}(\boldsymbol{\Phi})\right\|_{F}^{2},
$$

where $\boldsymbol{F}_{n, j t}^{(2)}(\boldsymbol{\Phi})$ and $\boldsymbol{F}_{n, j s t}^{(3)}(\boldsymbol{\Phi})$ are defined as

$$
\boldsymbol{F}_{n, j t}^{(2)}(\boldsymbol{\Phi})=\frac{1}{n} \sum_{i=1}^{n} \boldsymbol{F}_{j t}^{(2)}\left(\boldsymbol{y}_{i}, \boldsymbol{\Phi}\right), \quad \boldsymbol{F}_{n, j s t}^{(3)}(\boldsymbol{\Phi})=\frac{1}{n} \sum_{i=1}^{n} \boldsymbol{F}_{j s t}^{(3)}\left(\boldsymbol{y}_{i}, \boldsymbol{\Phi}\right),
$$

with $\boldsymbol{\mu}_{j}, \boldsymbol{\mu}_{s}$ and $\boldsymbol{\mu}_{t}$ replaced by $\widehat{\boldsymbol{\mu}}_{j}, \widehat{\boldsymbol{\mu}}_{s}$ and $\widehat{\boldsymbol{\mu}}_{t}$ respectively in $\boldsymbol{F}_{j t}^{(2)}\left(\boldsymbol{y}_{i}, \boldsymbol{\Phi}\right)$ and $\boldsymbol{F}_{j s t}^{(3)}\left(\boldsymbol{y}_{i}, \boldsymbol{\Phi}\right)$. $\|\cdot\| \|_{F}^{2}$ indicates the Frobenius norm, the element-wise sum of squares.

We obtain a first stage estimator of $\boldsymbol{\Phi}$ by minimizing the quadratic forms using Newton-Raphson. Note that after we substitute $\boldsymbol{\mu}_{j}$ by its sample estimate, only the last term of $\boldsymbol{F}_{n, j t}^{(2)}(\boldsymbol{\Phi})$ and $\boldsymbol{F}_{n, j s t}^{(3)}(\boldsymbol{\Phi})$ involves unknown parameter $\boldsymbol{\Phi}$. For simplicity we denote

$$
\begin{aligned}
\boldsymbol{E}_{n, j t}^{(2)} & =\boldsymbol{F}_{n, j t}^{(2)}(\boldsymbol{\Phi})+\boldsymbol{\Phi}_{j} \boldsymbol{\Lambda}^{(2)} \boldsymbol{\Phi}_{t}^{T} \\
\boldsymbol{E}_{n, j s t}^{(3)} & =\boldsymbol{F}_{n, j s t}^{(3)}(\boldsymbol{\Phi})+\boldsymbol{\Lambda}^{(3)} \times_{1} \boldsymbol{\Phi}_{j} \times_{2} \boldsymbol{\Phi}_{s} \times_{3} \boldsymbol{\Phi}_{t} .
\end{aligned}
$$

The two quantities $\boldsymbol{E}_{n, j t}^{(2)}$ and $\boldsymbol{E}_{n, j s t}^{(3)}$ can be computed directly from the samples. We optimize $\boldsymbol{\phi}_{j h}$ with other mean parameters fixed. After calculating the gradient and Hessian of $Q_{n}^{(2)}\left(\boldsymbol{\phi}_{j h}, \boldsymbol{I}\right)$, the update rule simply becomes

$$
\boldsymbol{\phi}_{j h}^{s}=\frac{\sum_{t=1, t \neq j}^{p}\left(\overline{\boldsymbol{E}}_{n, j t}^{(2)} \boldsymbol{\phi}_{t h}\right)^{T}}{\left(\lambda_{h}^{(2)}\right) \sum_{t=1, t \neq j}^{p} \boldsymbol{\phi}_{t h}^{T} \boldsymbol{\phi}_{t h}}
$$

where $\overline{\boldsymbol{E}}_{n, j t}^{(2)}=\boldsymbol{E}_{n, j t}^{(2)}-\sum_{h^{\prime} \neq h} \lambda_{h^{\prime}}^{(2)} \boldsymbol{\phi}_{j h^{\prime}} \circ \boldsymbol{\phi}_{t h^{\prime}}$ and $\lambda_{h}^{(2)}$ is the $h$ th diagonal entry of $\boldsymbol{\Lambda}^{(2)}$.

The update rule for $\boldsymbol{\phi}_{j h}$ with $Q_{n}^{(3)}\left(\boldsymbol{\phi}_{j h}, \boldsymbol{I}\right)$ can be calculated as

$$
\boldsymbol{\phi}_{j h}^{s}=\frac{\lambda_{h}^{(2)} \sum_{t=1, t \neq j}^{p}\left(\overline{\boldsymbol{E}}_{n, j t}^{(2)} \boldsymbol{\phi}_{t h}\right)^{T}+\lambda_{h}^{(3)} \sum_{s=1, s \neq j}^{p}\left[\sum_{t=1, t \neq s, t \neq j}^{p}\left(\overline{\boldsymbol{E}}_{n, j s t}^{(3)} \times_{2} \boldsymbol{\phi}_{s h} \times_{3} \boldsymbol{\phi}_{t h}\right)^{T}\right]}{\left(\lambda_{h}^{(2)}\right)^{2} \sum_{t=1, t \neq j}^{p} \boldsymbol{\phi}_{t h}^{T} \boldsymbol{\phi}_{t h}+\left(\lambda_{h}^{(3)}\right)^{2} \sum_{s=1, s \neq j}^{p}\left[\sum_{t=1, t \neq s, t \neq j}^{p}\left(\boldsymbol{\phi}_{s h}^{T} \boldsymbol{\phi}_{s h}\right)\left(\boldsymbol{\phi}_{t h}^{T} \boldsymbol{\phi}_{t h}\right)\right]}
$$

where $\overline{\boldsymbol{E}}_{n, j s t}^{(3)}=\boldsymbol{E}_{n, j s t}^{(3)}-\sum_{h^{\prime} \neq h} \lambda_{h^{\prime}}^{(3)} \boldsymbol{\phi}_{j h^{\prime}} \circ \boldsymbol{\phi}_{s h^{\prime}} \circ \boldsymbol{\phi}_{t h^{\prime}}$ and $\lambda_{h}^{(3)}$ is the $h$ th diagonal entry in $\boldsymbol{\Lambda}^{(3)}$. The derivations can be found in Appendix E. After updating $\phi_{j h}$ using the above equations, we retract $\phi_{j h}$ to its probability simplex when $y_{i j}$ is a categorical variable. We use the difference of the objective function between two iterations divided by the dimension of the moment vector to determine convergence. In particular, we stop iterations when this value is smaller than $1 \times 10^{-5}$.

After an initial consistent estimator of $\boldsymbol{\Phi}$ is found, we calculate the asymptotic covariance matrix of the two versions of moment vectors $\boldsymbol{S}_{n}^{(\cdot)}$ and define a new weight matrix $\boldsymbol{A}_{n}^{(\cdot)}=\left(\boldsymbol{S}_{n}^{(\cdot)}\right)^{-1}$ for a second stage GMM estimation. The form of $\boldsymbol{S}_{n}^{(\cdot)}$ can be derived analytically, and we provide the results in the Supplement Materials. In our implementation, the calculation of $\boldsymbol{A}_{n}^{(\cdot)}$ requires the inversion of a full-rank dense matrix $\boldsymbol{S}_{n}^{(\cdot)}$ with dimension scaling as $O\left(p^{2} d^{2}\right)$ for $\boldsymbol{f}_{n}^{(2)}(\boldsymbol{\Phi})$ and $O\left(p^{3} d^{3}\right)$ for $\boldsymbol{f}_{n}^{(3)}(\boldsymbol{\Phi})$. In addition, when including the off-diagonal terms in the weight matrix, the updating rules become intrinsically complicated. In practice, we only extract the diagonal elements of $\boldsymbol{S}_{n}^{(\cdot)}$ and let $\boldsymbol{A}_{n}^{(\cdot)}=1 / \operatorname{diag}\left[\left(\boldsymbol{S}_{n}^{(\cdot)}\right)\right]$ in the second stage estimation. This approximation has been used in previous GMM implementations (Jöreskog and Sörbom, 1987). The gradient descent update equations can be found by slight modification of Equations 11 and 12 with weights included. 
Note that the moment functions do not solve the identifiability problems with respect to $\boldsymbol{\Phi}$. When $\boldsymbol{\alpha}$ is a constant vector, any permutation $\tau$ of $1, \ldots, k$ with $\boldsymbol{\Phi}_{j}(\tau)=\left(\phi_{j \tau(1)}, \ldots, \phi_{j \tau(k)}\right)$ for all $j=1, \ldots, p$ satisfies the moment condition. This problem is inherited from the label switching problem in mixture models. A similar situation occurs when there are ties in $\boldsymbol{\alpha}$ and the permutation is restricted to each tie. However, in real applications, a minimizer of the quadratic function is generally sufficient to find a parameter estimate that is close to a unique configuration of the true parameter.

\subsection{Properties of parameter estimates}

We use GMM asymptotic theory to show that parameter estimates in MELD are consistent. We assume the following regularity conditions on the two versions of moment vector $\boldsymbol{f}^{(\cdot)}\left(\boldsymbol{y}_{i}, \boldsymbol{\Phi}\right)$ and the parameter space $\boldsymbol{\Theta}$.

Assumption 1 (Regularity conditions (Assumption 3.2, 3.9 and $3.10\left(\right.$ Hall, 2005))) 1) $\boldsymbol{f}^{(\cdot)}\left(\boldsymbol{y}_{i}, \boldsymbol{\Phi}\right)$ is continuous on $\boldsymbol{\Theta}$ for all $\boldsymbol{y}_{i} \in \mathcal{Y}$;2) $\mathbb{E}\left[\boldsymbol{f}^{(\cdot)}\left(\boldsymbol{y}_{i}, \boldsymbol{\Phi}\right)\right]<\infty$ and continuous for $\boldsymbol{\Phi} \in \boldsymbol{\Theta}$; 3) $\boldsymbol{\Theta}$ is compact and $\mathbb{E}\left[\sup _{\boldsymbol{\Phi} \in \boldsymbol{\Theta}}\left\|\boldsymbol{f}^{(\cdot)}\left(\boldsymbol{y}_{i}, \boldsymbol{\Phi}\right)\right\|\right]<\infty$.

Remark 1. Conditions 1) and 2) are satisfied in MELD. Condition 3) is satisfied automatically for categorical variables, noting that $\phi_{j h} \in \Delta^{d_{j}-1} \subset \mathbb{R}^{d_{j}}$ is compact. For non-categorical variables, we can restrict support to a large compact domain such as closed intervals in $\mathbb{R}$ without sacrificing practical performance.

With these conditions, we further assume that the weight matrix $\boldsymbol{A}_{n}^{(\cdot)}$ converges to a positive definite matrix $\boldsymbol{A}^{(\cdot)}$ in probability. We define the population analogs of the quadratic functions as

$$
\begin{aligned}
& Q_{0}^{(2)}\left(\boldsymbol{\Phi} ; \boldsymbol{A}^{(2)}\right)=\mathbb{E}\left[\boldsymbol{f}^{(2)}\left(\boldsymbol{y}_{i}, \boldsymbol{\Phi}\right)\right]^{T} \boldsymbol{A}^{(2)} \mathbb{E}\left[\boldsymbol{f}^{(2)}\left(\boldsymbol{y}_{i}, \boldsymbol{\Phi}\right)\right] \\
& Q_{0}^{(3)}\left(\boldsymbol{\Phi} ; \boldsymbol{A}^{(3)}\right)=\mathbb{E}\left[\boldsymbol{f}^{(3)}\left(\boldsymbol{y}_{i}, \boldsymbol{\Phi}\right)\right]^{T} \boldsymbol{A}^{(3)} \mathbb{E}\left[\boldsymbol{f}^{(3)}\left(\boldsymbol{y}_{i}, \boldsymbol{\Phi}\right)\right]
\end{aligned}
$$

We have the following lemma showing the uniform convergence of $Q_{n}^{(\cdot)}\left(\boldsymbol{\Phi} ; \boldsymbol{A}_{n}^{(\cdot)}\right)$.

Lemma 1 (Uniform convergence (Lemma 3.1 (Hall, 2005))). Under regularity conditions in Assumption 1.

$$
\sup _{\mathbf{\Phi} \in \boldsymbol{\Theta}}\left|Q_{n}^{(2)}\left(\boldsymbol{\Phi} ; \boldsymbol{A}_{n}^{(2)}\right)-Q_{0}^{(2)}\left(\boldsymbol{\Phi} ; \boldsymbol{A}^{(2)}\right)\right| \stackrel{p}{\rightarrow} 0 \quad \sup _{\boldsymbol{\Phi} \in \boldsymbol{\Theta}}\left|Q_{n}^{(3)}\left(\boldsymbol{\Phi} ; \boldsymbol{A}_{n}^{(3)}\right)-Q_{0}^{(3)}\left(\boldsymbol{\Phi} ; \boldsymbol{A}^{(3)}\right)\right| \stackrel{p}{\rightarrow} 0 .
$$

Theorem 2 (Consistency). Under the same conditions in Lemma 1, the estimator $\widehat{\boldsymbol{\Phi}}^{(2)}$ that minimizes $Q_{n}^{(2)}\left(\boldsymbol{\Phi} ; \boldsymbol{A}_{n}^{(2)}\right)$ converges to the true parameter $\boldsymbol{\Phi}_{0}$ in probability. A similar result holds for $\widehat{\boldsymbol{\Phi}}^{(3)}$ that minimizes $Q_{n}^{(3)}\left(\boldsymbol{\Phi} ; \boldsymbol{A}_{n}^{(3)}\right)$.

The proof can be found in Appendix B

The asymptotic normality of $\widehat{\boldsymbol{\Phi}}^{(2)}$ and $\widehat{\boldsymbol{\Phi}}^{(3)}$ can also be established by assuming the following conditions on $\partial \boldsymbol{f}^{(\cdot)}\left(\boldsymbol{y}_{i}, \boldsymbol{\Phi}\right) / \partial \boldsymbol{\Phi}$.

Assumption 2 (Conditions on $\partial \boldsymbol{f}^{(\cdot)}\left(\boldsymbol{y}_{i}, \boldsymbol{\Phi}\right) / \partial \boldsymbol{\Phi}$ (Assumptions 3.5, 3.12 and 3.13. Hall, 2005)) . 1) $\partial \boldsymbol{f}^{(\cdot)}\left(\boldsymbol{y}_{i}, \boldsymbol{\Phi}\right) / \partial \boldsymbol{\Phi}$ exists and is continuous on $\boldsymbol{\Theta}$ for all $\left.\boldsymbol{y}_{i} \in \mathcal{Y} ; 2\right) \boldsymbol{\Phi}_{0}$ is an interior point of $\left.\boldsymbol{\Theta} ; 3\right) \mathbb{E}\left[\partial \boldsymbol{f}^{(\cdot)}\left(\boldsymbol{y}_{i}, \boldsymbol{\Phi}\right) / \partial \boldsymbol{\Phi}\right]=\boldsymbol{G}_{0}^{(\cdot)}(\boldsymbol{\Phi})<\infty$; 4) $\boldsymbol{G}_{0}^{(\cdot)}(\boldsymbol{\Phi})$ is continuous on some neighborhood $N_{\epsilon}$ of $\left.\boldsymbol{\Phi}_{0} ; 5\right)$ the sample estimate $\boldsymbol{G}_{n}^{(\cdot)}(\boldsymbol{\Phi})$ uniformly converges to $\boldsymbol{G}_{0}^{(\cdot)}(\boldsymbol{\Phi})$. 
Remark 2. We derive $\partial \boldsymbol{f}^{(\cdot)}\left(\boldsymbol{y}_{i}, \boldsymbol{\Phi}\right) / \partial \boldsymbol{\Phi}$ in Appendix D. Conditions 1, 3, and 4 are satisfied in MELD. Condition 5 can be shown with continuousness of the derivative and the compactness of $\boldsymbol{\Theta}$.

Theorem 3 (Asymptotic normality). With Assumptions 1 and 2, we have

$$
n^{1 / 2}\left(\operatorname{vec}\left(\widehat{\boldsymbol{\Phi}}^{(\cdot)}\right)-\operatorname{vec}\left(\boldsymbol{\Phi}_{0}\right)\right) \stackrel{p}{\rightarrow} \mathcal{N}\left(\mathbf{0}, \boldsymbol{M}^{(\cdot)} \boldsymbol{S}^{(\cdot)}\left(\boldsymbol{M}^{(\cdot)}\right)^{T}\right)
$$

with

$$
\boldsymbol{M}^{(\cdot)}=\left[\left(\boldsymbol{G}_{0}^{(\cdot)}\right)^{T} \boldsymbol{A}^{(\cdot)} \boldsymbol{G}_{0}^{(\cdot)}\right]^{-1}\left(\boldsymbol{G}_{0}^{(\cdot)}\right)^{T} \boldsymbol{A}^{(\cdot)},
$$

where $\boldsymbol{G}_{0}^{(\cdot)}=\left.\mathbb{E}\left[\partial \boldsymbol{f}^{(\cdot)}\left(\boldsymbol{y}_{i}, \boldsymbol{\Phi}\right) / \partial \boldsymbol{\Phi}\right]\right|_{\boldsymbol{\Phi}=\boldsymbol{\Phi}_{0}}$ and $\boldsymbol{S}^{(\cdot)}=\lim _{n \rightarrow \infty} \operatorname{Var}\left[n^{1 / 2} \boldsymbol{f}_{n}^{(\cdot)}\left(\boldsymbol{\Phi}_{0}\right)\right]$. The proof can be found in Appendix C. The optimal estimator can be obtained so that the weight matrix $\boldsymbol{A}_{n}^{(\cdot)} \rightarrow \boldsymbol{A}^{(\cdot)}=\left(\boldsymbol{S}^{(\cdot)}\right)^{-1}$ (Hansen, 1982).

\subsection{Model selection using goodness of fit tests}

We use goodness of fit tests to choose the number of latent components $k$. With the optimal weight matrix $\boldsymbol{A}_{n}^{(\cdot)} \rightarrow\left(\boldsymbol{S}^{(\cdot)}\right)^{-1}$, Wald-, Lagrange multiplier- (score) and likelihood ratio-type tests can be constructed. We could construct a sequence of test statistics with increasing $k$ to quantify the goodness of fit in MELD. However, this approach requires the calculation of the optimal weight matrix and large matrix inversions. Instead, we quantify goodness of fit using a fitness index (FI) (Bentler, 1983). Practically, we show in an extensive simulation study that the FI has low error in estimating $k$ in MELD.

The FI is defined based on the value of the objective function evaluated at the parameter estimate (Bentler, 1983)

$$
\mathrm{FI}=1-\frac{Q_{n}^{(\cdot)}\left(\widehat{\boldsymbol{\Phi}}^{(\cdot)}, \boldsymbol{A}_{n}^{(\cdot)}\right)}{\left(\boldsymbol{e}_{n}^{(\cdot)}\right)^{T} \boldsymbol{A}_{n}^{(\cdot)} \boldsymbol{e}_{n}^{(\cdot)}}
$$

where $\boldsymbol{e}_{n}^{(2)}$ is the concatenation of $\operatorname{vec}\left(\boldsymbol{E}_{n, j t}^{(2)}\right)$ for $j<t$ and $\boldsymbol{e}_{n}^{(3)}$ is the concatenation of both $\operatorname{vec}\left(\boldsymbol{E}_{n, j t}^{(2)}\right)$ for $j<t$ and $\operatorname{vec}\left(\boldsymbol{E}_{n, j s t}^{(3)}\right)$ for $j<s<t$. This FI is for any weight matrix $\boldsymbol{A}_{n}^{(\cdot)}$. It can be viewed as a normalized objective function; thus, the FI has values less than one. Larger values of FI indicate a better fit.

\section{Simulation study}

In this section, we evaluate the accuracy and run time of MELD in simulations with both categorical and mixed data types. We use two stage estimations described in previous sections. In the first stage an identity weight matrix is used. In second stage we set $\boldsymbol{A}_{n}^{(\cdot)}=1 / \operatorname{diag}\left[\left(\boldsymbol{S}_{n}^{(\cdot)}\right)\right]$. For notation convenience we suppress the weight matrix $\boldsymbol{A}^{(\cdot)}$ and the subscript $n$ in the objective functions $Q_{n}^{(\cdot)}\left(\boldsymbol{\Phi}, \boldsymbol{A}^{(\cdot)}\right)$.

\subsection{Categorical data}

For simulation with categorical data, we considered a low dimensional setting $(p=20)$ so that both second and third order moment functions may be efficiently calculated. A simulation with a moderate dimension $(p=100)$ was also studied and is summarized in the Supplementary Materials.

We assumed each of the 20 variables has $d=4$ levels. We set the number of components to $k=3$ and generated $\phi_{j h}$ from $\operatorname{Dir}(0.5,0.5,0.5,0.5)$ with $h=1, \ldots, k$, and $\boldsymbol{\alpha}$ was set to $(0.1,0.1,0.1)^{T}$. We 
drew $n=\{50,100,200,500,1,000\}$ samples from the generative model in Equation (1) with $g_{j}\left(\phi_{j h}\right)$ a multinomial distribution. For each value of $n$, we generated ten independent data sets. We ran MELD for different values of $k=\{1, \ldots, 5\}$. The FI criterion consistently chose the correct number of latent components $k$ in first stage estimation (Table 1). For second stage, FI did not perform well. The trajectories of the objective functions under different values of $k$ are shown in Figure S1. The convergence of parameter estimations on the ten simulated data sets under $n=1,000$ and $k=3$ is shown in Figure S2. MELD converged in about 25 iterations with $Q^{(2)}(\boldsymbol{\Phi})$ and in about 10 iterations with $Q^{(3)}(\boldsymbol{\Phi})$.

Table 1: Goodness of fit tests using the fitness index (FI) in categorical simulation. Larger values of FI indicate better fit, with the maximum at one. Results shown are based on ten simulated data sets for each value of $n$. Standard deviations of FI are provided in parentheses.

\begin{tabular}{|c|c|c|c|c|c|}
\hline$n$ & $k$ & $Q^{(2)}(\Phi)$ 1st stage & $Q^{(2)}(\Phi)$ 2nd stage & $Q^{(3)}(\Phi)$ 1st stage & $Q^{(3)}(\Phi)$ 2nd stage \\
\hline \multirow{5}{*}{50} & 1 & $0.824(0.020)$ & $-1.865(4.950)$ & $0.585(0.031)$ & $-27.987(40.128)$ \\
\hline & 2 & $0.908(0.011)$ & $-1.285(2.630)$ & $0.745(0.032)$ & $-27.240(54.415)$ \\
\hline & 3 & $0.930(0.004)$ & $0.570(0.044)$ & $0.775(0.022)$ & $-27.713(42.607)$ \\
\hline & 4 & $0.901(0.010)$ & $0.588(0.032)$ & $0.735(0.023)$ & $0.254(0.090)$ \\
\hline & 5 & $0.795(0.033)$ & $0.686(0.021)$ & $0.653(0.024)$ & $0.305(0.091)$ \\
\hline \multirow{5}{*}{100} & 1 & $0.860(0.012)$ & $-0.521(2.142)$ & $0.651(0.021)$ & $-0.031(0.703)$ \\
\hline & 2 & $0.930(0.011)$ & $0.282(0.644)$ & $0.795(0.030)$ & $-4.457(8.924)$ \\
\hline & 3 & $0.960(0.005)$ & $0.677(0.044)$ & $0.851(0.009)$ & $-4.868(11.889)$ \\
\hline & 4 & $0.942(0.012)$ & $0.691(0.042)$ & $0.822(0.010)$ & $0.225(0.019)$ \\
\hline & 5 & $0.863(0.046)$ & $0.782(0.022)$ & $0.768(0.044)$ & $0.232(0.080)$ \\
\hline \multirow{5}{*}{200} & 1 & $0.869(0.012)$ & $0.679(0.060)$ & $0.682(0.021)$ & $0.298(0.070)$ \\
\hline & 2 & $0.940(0.007)$ & $0.699(0.047)$ & $0.838(0.014)$ & $0.306(0.054)$ \\
\hline & 3 & $0.980(0.001)$ & $0.761(0.061)$ & $0.919(0.004)$ & $0.278(0.049)$ \\
\hline & 4 & $0.967(0.006)$ & $0.780(0.019)$ & $0.891(0.008)$ & $0.287(0.050)$ \\
\hline & 5 & $0.911(0.017)$ & $0.824(0.008)$ & $0.864(0.015)$ & $0.286(0.042)$ \\
\hline \multirow{5}{*}{500} & 1 & $0.882(0.007)$ & $0.783(0.022)$ & $0.713(0.012)$ & $0.414(0.080)$ \\
\hline & 2 & $0.948(0.006)$ & $0.799(0.019)$ & $0.870(0.013)$ & $0.427(0.080)$ \\
\hline & 3 & $0.992(<0.001)$ & $0.884(0.024)$ & $0.966(0.001)$ & $0.388(0.073)$ \\
\hline & 4 & $0.983(0.004)$ & $0.874(0.026)$ & $0.938(0.005)$ & $0.365(0.065)$ \\
\hline & 5 & $0.937(0.014)$ & $0.894(0.006)$ & $0.921(0.007)$ & $0.353(0.042)$ \\
\hline \multirow{5}{*}{1,000} & 1 & $0.888(0.003)$ & $0.828(0.006)$ & $0.729(0.008)$ & $0.571(0.017)$ \\
\hline & 2 & $0.951(0.003)$ & $0.855(0.009)$ & $0.881(0.008)$ & $0.615(0.030)$ \\
\hline & 3 & $0.996(<0.001)$ & $0.950(0.005)$ & $0.982(0.001)$ & $0.609(0.031)$ \\
\hline & 4 & $0.989(0.002)$ & $0.938(0.004)$ & $0.961(0.003)$ & $0.579(0.030)$ \\
\hline & 5 & $0.953(0.010)$ & $0.932(0.006)$ & $0.951(0.006)$ & $0.550(0.034)$ \\
\hline
\end{tabular}

We compared MELD with the simplex factor model (SFM) (Bhattacharya and Dunson, 2012) and latent Dirichlet allocation (LDA) (Blei et al., 2003). For SFM, we ran 10,000 steps of MCMC with fixed $k$ and a burn-in of 5,000 iterations. Posterior thinned samples were collected by keeping one posterior draw after every 50 steps. For the LDA model, we used the lda package in R (Chang, 2012) with collapsed Gibbs sampling. We used the same number of MCMC iterations and burn-in iterations as with SFM. The Dirichlet parameter for mixture proportions was set to $\alpha=0.1$ and the Dirichlet parameter for topic distributions was set to $\beta=0.5$. We calculated mean squared errors (MSE's) of different estimates as follows. We first recovered the membership variable $m_{i j}$ and then calculated the parameter estimate of $y_{i j}$. The MSE was calculated between the estimated parameters of $y_{i j}$ 's and their true parameters (see Supplementary Materials for details). MSE's with different values of $k$ are shown in Figure 1 and the running times of different methods are reported in Table S1. MELD $Q^{(2)}(\boldsymbol{\Phi})$ with first stage estimation had the most accurate parameter estimation and fastest running speed in most cases. The second stage of $\operatorname{MELD} Q^{(3)}(\boldsymbol{\Phi})$ did not perform well with small values of $n$ (i.e., $n=50$ ), but estimation accuracy was better when $n$ was larger. SFM had comparable MSE's when $n$ was not large. However when $n=500$ and 1,000, MELD outperformed SFM.

We further evaluated performance in the presence of contamination. For each simulated data set, 
we randomly replaced a proportion of observations (4\% and $10 \%$ ) with draws from a discrete uniform distribution. The MSE's under different values of $k$ are shown in Figure 1 . With $4 \%$ contamination, MELD had the most accurate parameter estimation in almost all cases. MELD $Q^{(2)}(\boldsymbol{\Phi})$ with first stage estimation performed the best, followed by $\operatorname{MELD} Q^{(3)}(\boldsymbol{\Phi})$ with first stage estimation. The MSE's of SFM increased. When we increased contamination to $10 \%$, MELD had the most accurate MSE in all cases. MELD $Q^{(2)}(\boldsymbol{\Phi})$ with first stage estimation performed best, followed by MELD $Q^{(2)}(\boldsymbol{\Phi})$ with second stage estimation. MELD $Q^{(2)}(\boldsymbol{\Phi})$ consistently performed better than $Q^{(3)}(\boldsymbol{\Phi})$, suggesting the robustness of using lower order moments in parameter estimation.
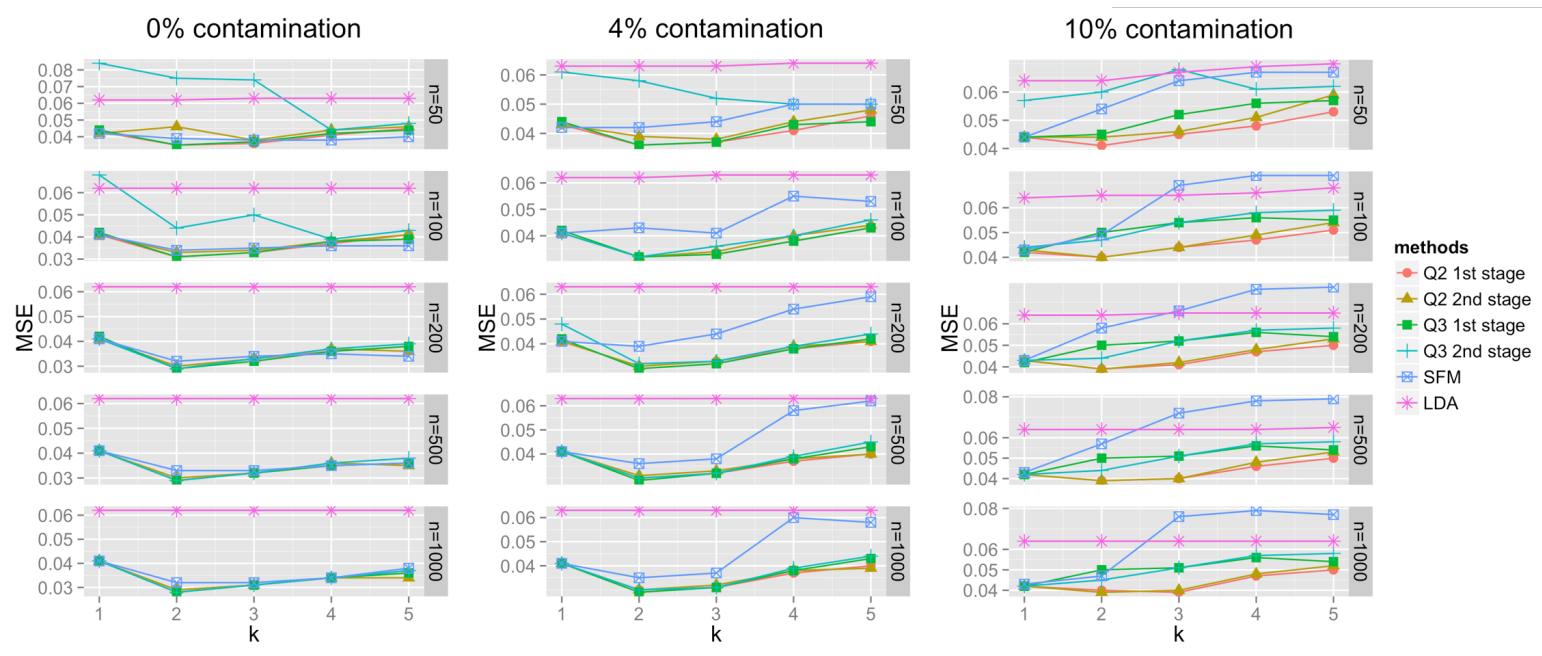

Figure 1: Comparison of mean squared error (MSE) of estimated parameters in categorical simulations. For SFM and LDA, posterior means of parameters are calculated using 100 posterior draws on each of the ten simulated data sets. The values of the MSE's and their standard deviations are in Supplementary Table S2, S3 and S4.

\subsection{Mixed data types}

We considered a simulation setting mimicking applications in which DNA sequence variations influence a quantitative trait. An additional simulation combining categorical, Gaussian, and Poisson variables is summarized in the Supplementary Materials. We generated a sequence of nucleotides $\{A, C, G, T\}$ at 50 genetic loci along with a continuous or integer-valued trait, leading to $p=51$ variables. We set $k=2$ latent components and simulated $n=1,000$ individuals, with the first 500 from the first component and the last 500 from the second component. We chose eight loci $J=\{2,4,12,14,32,34,42,44\}$ to be associated with the trait. Their multinomial parameters for each of the two components were randomly drawn from $\operatorname{Dir}(0.5,0.5,0.5,0.5)$. The distributions for nucleotides in other loci were set to $\operatorname{Multi}(0.25,0.25,0.25,0.25)$. Continuous traits were drawn from $\mathcal{N}(-3,1)$ and $\mathcal{N}(3,1)$, while count traits were drawn from Poisson(5) and Poisson(10), respectively for the two components. Ten data sets were simulated from the generative model in Equation (1). To assess robustness, we added contamination (e.g., through genotyping errors) by randomly replacing 4\%, $10 \%$ and $20 \%$ of the nucleotides with values uniformly generated from $\{A, C, G, T\}$.

We ran MELD with first stage estimation of $Q^{(2)}(\boldsymbol{\Phi})$. We chose the number of components $k=$ $\{1, \ldots, 5\}$. The fitness test indicated that FI chose the correct value of $k$ on all ten data sets (Table S8). 
For each genomic locus, we calculated its marginal frequency according to the simulated data, and then we computed the averaged KL distance between the estimated component distributions and the marginal frequency as follows

$$
\operatorname{aveKL}\left(y_{i j}\right)=\frac{1}{k} \sum_{h=1}^{k} \sum_{c_{j}=1}^{d_{j}} \operatorname{Pr}\left(y_{i j}=c_{j} \mid m_{i j}=h\right) \log \left(\frac{\operatorname{Pr}\left(y_{i j}=c_{j} \mid m_{i j}=h\right)}{\operatorname{Pr}\left(y_{i j}=c_{j}\right)}\right) .
$$

A smaller averaged KL distance suggests that the component distributions were closer to the marginal distribution, implying that the locus frequency was not differentiated across components. The set $J$ corresponded exact to the eight loci with largest averaged KL distance (Table 2).

We compared MELD with the Bayesian copula factor model (Murray et al., 2013), which estimates a correlation matrix $\boldsymbol{C}$ between variables. From this estimate, we computed partial correlations between the response variable (trait) and each genetic locus (Hoff, 2007). We ran MCMC for the Bayesian copula factor model 10,000 iterations with the correct value for $k$ and a burn-in of 5,000 iterations. Posterior samples were collected every 50 iterations. We then selected genomic locations for which their $95 \%$ posterior intervals of the partial correlation did not include zero. The resulting loci are shown in Table 2 The Bayesian copula factor model selected nucleotides that are not in $J$ and missed locus 32 in most cases.

Table 2: Quantitative trait association simulation with 50 nucleotides and one response. Nucleotides not in $J=\{2,4,12,14,32,34,42,44\}$ are labeled by an underline and missing nucleotides are crossed out. Results shown are for one of the ten simulated data sets. The complete results can be found in Table S9 and S10.

\begin{tabular}{|c|c|c|c|}
\hline Response & Contamination & $Q^{(2)}(\boldsymbol{\Phi})$ 1st stage & Bayesian copula factor model \\
\hline \multirow{4}{*}{ Gaussian } & $0 \%$ & $\{2,4,12,14,32,34,42,44\}$ & $\{2,4,12,14, \underline{18}, \underline{27}, 322,34,42,44\}$ \\
\hline & $4 \%$ & $\{2,4,12,14,32,34,42,44\}$ & $\{2,4,12,14, \underline{18}, \underline{27}, 322,34,42,44, \underline{45}\}$ \\
\hline & $10 \%$ & $\{2,4,12,14,32,34,42,44\}$ & $\{2,4,12,14, \underline{27}, \not 22,34,42,44, \underline{49}, \underline{50}\}$ \\
\hline & $20 \%$ & $\{2,4,12,14,32,34,42,44\}$ & $\{2,4, \underline{9}, \underline{11}, 12,14, \underline{20}, \not 22,34,42,44\}$ \\
\hline \multirow{4}{*}{ Poisson } & $0 \%$ & $\{2,4,12,14,32,34,42,44\}$ & $\{2,4, \underline{7}, 12,14,322,34,42,44\}$ \\
\hline & $4 \%$ & $\{2,4,12,14,32,34,42,44\}$ & $\{2,4,12,14, \not 22,34,42,44\}$ \\
\hline & $10 \%$ & $\{2,4,12,14,32,34,42,44\}$ & $\{2,4, \underline{7}, 12,14, \underline{16}, 32,34,42,44\}$ \\
\hline & $20 \%$ & $\{2,4,12,14,32,34,42,44\}$ & $\{2,4, \underline{7}, 12,14,32,34, \underline{35}, 42,44\}$ \\
\hline
\end{tabular}

\section{Applications of MELD}

Promoter sequence analysis We applied MELD to gene transcription promoter data available in the UCI machine learning repository (Lichman, 2013). The data include $n=106$ nucleotide sequences $\{A, C, G, T\}$ of length 57 . The first 53 sequences are located in promoter regions, and the last 53 sequences are located in non-promoter regions. The goal is binary classification: using nucleotide sequence alone, we would like to predict whether or not the sequence is in a promoter or a non-promoter region.

We included the promoter or non-promoter status of the sequences as an additional binary variable, giving us $p=57+1$ categorical variables. We applied MELD $Q^{(2)}(\boldsymbol{\Phi})$ with first stage estimation on the full data and also on the subset of the sequences in the promoter region and the subset of sequences in non-promoter regions separately. We set $k=\{1, \ldots, 8\}$. For $k=2$, MELD converged in 2.13 seconds, compared with SFA, which took 41.6 seconds to perform 10, 000 MCMC iterations. We evaluated different 
values of $k$ using the goodness of fit test. FI selected two components for the full data, two components for the promoter data, and one component for the non-promoter data (Table S13).

We choose $k=2$ in the following analysis. For each nucleotide position, we calculated the averaged KL distance between the estimated component distributions and its marginal distribution using Equation (16). An interpretation of the averaged KL distance is that it quantifies the stratification of each nucleotide distribution across components: a larger value of the averaged KL distance indicates greater stratification across components, which suggests that the nucleotide is important in defining and differentiating the components. For the full data set, we observed approximately two peaks of the averaged KL distance, one around the 15th nucleotide and one around the 42nd nucleotide (Figure S4). The first peak corresponds to the start of the biologically conserved region for promoter sequences (Harley and Reynolds, 1987). For MELD applied only to promoter sequences, this peak was reduced, suggesting that, at approximately the 15th nucleotide, the components all included similarly well conserved distributions of this nucleotide. The estimated component membership variables also showed the importance of nucleotides around 15th nucleotide (Figure S5 and S6). For the peak around the 42nd nucleotide, this phenomenon was reversed: the increased averaged KL distance remained in promoter sequences but diminished in non-promoter sequences. One possible explanation is that this region was conserved uniformly in the non-promoter region, but differentially conserved in the promoter region.

Political-economic risk data In a second application, we applied MELD to political-economic risk data (Quinn, 2004), which include five proxy variables of mixed types measured for 62 countries. The data set has been collected and analyzed to quantify a sense of political-economic risk, a latent quantity associated with each of the 62 countries, using a mixed Gaussian and probit factor model (Quinn, 2004) and a Bayesian copula factor model (Murray et al., 2013). The data are available in the MCMCpack package. There are three categorical variables and two real valued variables (Table 3 .

Table 3: Variables in the political-economic risk data

\begin{tabular}{|lll|}
\hline Variable & Type & Explanation \\
\hline ind.jud & binary & $\begin{array}{l}\text { An indicator variable that measures the independence } \\
\text { of the national judiciary. This variable is equal to one if the } \\
\text { judiciary is judged to be independent and equal to zero otherwise. }\end{array}$ \\
& & Black-market premium measurement. Original values are measured \\
blk.mkt & real & $\begin{array}{l}\text { as the black-market exchange rate (local currency per dollar) } \\
\text { divided by the official exchange rate minus one. Quinn }\end{array}$ \\
& transformed the original data to log scale. \\
\hline lack.exp.risk & ordinal & $\begin{array}{l}\text { Lack of appropriation risk measurement. } \\
\text { Six levels with coding } 0<1<2<3<4<5 .\end{array}$ \\
\hline lack.corrup & ordinal & $\begin{array}{l}\text { Lack of corruption measurement. } \\
\text { Six levels with coding } 0<1<2<3<4<5 .\end{array}$ \\
\hline gdp.worker & real & $\begin{array}{l}\text { Real gross domestic product }(\mathrm{GDP}) \text { per worker in } 1985 \text { international prices. } \\
\text { Recorded data are log transformed. }\end{array}$ \\
\hline
\end{tabular}

We applied MELD with $k=\{1, \ldots, 5\}$ using both $Q^{(2)}(\boldsymbol{\Phi})$ and $Q^{(3)}(\boldsymbol{\Phi})$ with first stage estimation to the data set. For $Q^{(2)}(\boldsymbol{\Phi})$ with $k=3$ MELD converged in $0.10 \mathrm{~s}$, and for $Q^{(3)}(\boldsymbol{\Phi})$ with $k=3$ MELD converged in $0.45 \mathrm{~s}$. The Bayesian copula factor model took 0.91s to complete 10,000 MCMC iterations. 
The FI criterion for $Q^{(2)}(\boldsymbol{\Phi})$ selected $k=4$ and, for $Q^{(3)}(\boldsymbol{\Phi})$, selected $k=3$ (Table S14). We chose results from $Q^{(3)}(\boldsymbol{\Phi})$ with $k=3$ for further analysis.

The estimated component parameters for the five variables showed distinct interpretations of the three components (Figure S7). We might interpret the three components as low-risk, intermediate-risk, and high-risk political-economic status respectively. The first component had a high probability of independence of the national judiciary (ind.jud being one) and a low measurement of black-market premium. The first component also had a high probability of observing 4th and 5th levels in lack.exp.risk and $3 \mathrm{rd}, 4 \mathrm{th}$, and 5th levels in lack. corrup. The mean of the GDP per worker was highest among the three components. The second component had a relatively high probability of being zero in ind.jud and a large mean value of blk.mkt. Both of lack of appropriation risk measurement and lack of corruption measurement put higher weights on lower category numbers (0,1 and 2), indicating more risk and higher levels of corruption; the GDP per worker was still high. We might interpret this component as a society being relatively unstable while still having a good economic forecast, meaning that GDP per worker is high, possibly through the black market. The last component had the least judicial independence as quantified by the probability of ind.jud being zero. The black-market premium is also low, as is the lack of risk level and lack of corruption level. The GDP per worker is by far the lowest among the three components. We might interpret this component as society being the most unstable with the greatest economic risk. We found although the three components had distinct stratification, each country was a mixture of the three components (Figure S8).

\section{Discussion}

In this paper, we developed a new class of latent variable models with Dirichlet-distributed latent variables for mixed data types. These generalized latent Dirichlet variable models extend previous mixed membership models such as LDA (Blei et al., 2003) and simplex factor models (Bhattacharya and Dunson, 2012) to allow mixed data types. For this class of models, we developed a fast parameter estimation procedure using generalized method of moments. Our procedure extends the moment tensor methods developed in recent work (Anandkumar et al. 2014b) to models with mixed data types. Our approach does not require instantiation of latent variables. We derive population moment conditions after marginalizing out the latent Dirichlet variables. We demonstrated the utility of our approach using simulations and two applications. Our results show that MELD is a promising alternative to MCMC or EM methods for parameter estimation, producing fast and robust parameter estimates. Since our method depends only on certain forms of sample moments, parameter estimation does not scale with sample size $n$ after the moment statistics have been computed from observations. An online method to update moment statistics when new samples arrive would allow re-estimation of the parameters to include new observations. One limitation of our method is that the Newton-Raphson method is of order $O\left(p^{2}\right)$ using second moment functions and order $O\left(p^{3}\right)$ using third moment functions. One possible approach to ensure tractability of MELD when $p$ is large is to use stochastic gradient methods to calculate an approximate gradient in each step.

\section{SUPPLEMENTARY MATERIALS}


We provide Supplementary Materials that include a) Derivations of asymptotic optimal weight matrices; b) Calculation of MSE and computational complexity analysis; c) Two additional simulations with categorical variables and mixed data types; d) Supplementary Tables S1-S14; e) Supplementary Figures $\mathrm{S} 1-\mathrm{S} 8$.

\section{Appendix}

\section{A Proof of Theorem 1}

Proof. We start with the case where $y_{i j}$ is a categorical data with $d_{j}$ different levels. The latent probability vector $\boldsymbol{x}_{i}=\left(x_{i 1}, \cdots, x_{i k}\right)^{T} \in \Delta^{k-1}$ defines the mixture proportion of individual $i$. We assume $\boldsymbol{x}_{i} \sim$ $\operatorname{Dir}\left(\alpha_{1}, \cdots, \alpha_{k}\right)$. Define $\alpha_{0}=\sum_{h} \alpha_{h}$ and $\boldsymbol{\alpha}=\left(\alpha_{1}, \cdots, \alpha_{k}\right)^{T}$.

We use the standard basis for encoding. We encode $y_{i j}=c_{j}$ as $\boldsymbol{b}_{i j} \in \mathbb{R}^{d_{j}}$ a binary (0/1) vector with the $c_{j}$ th coordinate being 1 and all others being 0. Similarly, we encode the membership variable $m_{i j}$ as a $k$ dimensional binary vector $\boldsymbol{m}_{i j} \in \mathbb{R}^{k}$. Consider the first moment of $\boldsymbol{b}_{i j}$.

$$
\boldsymbol{\mu}_{j}=\mathbb{E}\left(\boldsymbol{b}_{i j}\right)=\mathbb{E}\left[\mathbb{E}\left(\boldsymbol{b}_{i j} \mid \boldsymbol{m}_{i j}\right)\right]=\mathbb{E}\left(\boldsymbol{\Phi}_{j} \boldsymbol{m}_{i j}\right)=\mathbb{E}\left[\mathbb{E}\left(\boldsymbol{\Phi}_{j} \boldsymbol{m}_{i j} \mid \boldsymbol{x}_{i}\right)\right]=\mathbb{E}\left(\boldsymbol{\Phi}_{j} \boldsymbol{x}_{i}\right)=\boldsymbol{\Phi}_{j} \frac{\boldsymbol{\alpha}}{\alpha_{0}},
$$

where $\boldsymbol{\Phi}_{j}=\left(\phi_{j 1}, \cdots \phi_{j k}\right)$.

We consider second order moment conditions. There are four types of second moments: same variable same subject (type SS), same variable cross subject (type SC), cross variable same subject (type CS), and cross variable cross subject (type CC). Of the four types, only the CS type is needed to prove the theorem. The CS type second moment for $\boldsymbol{b}_{i j}$ and $\boldsymbol{b}_{i t}(j \neq t)$ can be written as

$$
\mathbb{E}\left(\boldsymbol{b}_{i j} \circ \boldsymbol{b}_{i t}\right)=\boldsymbol{\Phi}_{j} \mathbb{E}\left(\boldsymbol{m}_{i j} \circ \boldsymbol{m}_{i t}\right) \boldsymbol{\Phi}_{t}^{T}=\boldsymbol{\Phi}_{j} \mathbb{E}\left(\boldsymbol{x}_{i} \circ \boldsymbol{x}_{i}\right) \boldsymbol{\Phi}_{t}^{T}
$$

For a Dirichlet distributed variable,

$$
\begin{aligned}
\mathbb{E}\left(\boldsymbol{x}_{i} \circ \boldsymbol{x}_{i}\right) & =\operatorname{cov}\left(\boldsymbol{x}_{i}\right)+\mathbb{E}\left(\boldsymbol{x}_{i}\right) \circ \mathbb{E}\left(\boldsymbol{x}_{i}\right) \\
& =\frac{1}{\alpha_{0}\left(\alpha_{0}+1\right)} \operatorname{diag}(\boldsymbol{\alpha})+\frac{\alpha_{0}}{\alpha_{0}^{2}\left(\alpha_{0}+1\right)} \boldsymbol{\alpha} \circ \boldsymbol{\alpha} .
\end{aligned}
$$

Then we have

$$
\begin{aligned}
\mathbb{E}\left(\boldsymbol{b}_{i j} \circ \boldsymbol{b}_{i t}\right)=\boldsymbol{\Phi}_{j} \mathbb{E}\left(\boldsymbol{x}_{i} \circ \boldsymbol{x}_{i}\right) \boldsymbol{\Phi}_{t}^{T} \\
\quad=\frac{1}{\alpha_{0}\left(\alpha_{0}+1\right)} \sum_{h=1}^{k} \alpha_{h} \boldsymbol{\phi}_{j h} \circ \boldsymbol{\phi}_{t h}+\frac{\alpha_{0}}{\alpha_{0}+1} \boldsymbol{\mu}_{j} \circ \boldsymbol{\mu}_{t} .
\end{aligned}
$$

We next consider third order moment conditions. There are eight different types of third order moments for $\boldsymbol{b}_{i j}$. Only the moments with different variables for the same subject are needed to prove the theorem.

We consider the third cross moment for $\boldsymbol{b}_{i j}, \boldsymbol{b}_{i t}$ and $\boldsymbol{b}_{i s}$ with $j \neq t \neq s$ for the same subject. First we calculate $\mathbb{E}\left(\boldsymbol{m}_{i j} \circ \boldsymbol{m}_{i s} \circ \boldsymbol{m}_{i t}\right)$.

$$
\begin{aligned}
& \mathbb{E}\left(\boldsymbol{m}_{i j} \circ \boldsymbol{m}_{i s} \circ \boldsymbol{m}_{i t}\right)=\mathbb{E}\left[\mathbb{E}\left(\boldsymbol{m}_{i j} \circ \boldsymbol{m}_{i s} \circ \boldsymbol{m}_{i t} \mid \boldsymbol{x}_{i}\right)\right] \\
& \quad=\mathbb{E}\left(\boldsymbol{x}_{i} \circ \boldsymbol{x}_{i} \circ \boldsymbol{x}_{i}\right) \\
& \quad=\frac{1}{\alpha_{0}\left(\alpha_{0}+1\right)\left(\alpha_{0}+2\right)}\left((\boldsymbol{\alpha} \circ \boldsymbol{\alpha} \circ \boldsymbol{\alpha})+\sum_{h=1}^{k} \alpha_{h}\left(\boldsymbol{e}_{h} \circ \boldsymbol{e}_{h} \circ \boldsymbol{\alpha}\right)\right.
\end{aligned}
$$




$$
\begin{aligned}
& +\sum_{h=1}^{k} \alpha_{h}\left(\boldsymbol{e}_{h} \circ \boldsymbol{\alpha} \circ \boldsymbol{e}_{h}\right)+\sum_{h=1}^{k} \alpha_{h}\left(\boldsymbol{\alpha} \circ \boldsymbol{e}_{h} \circ \boldsymbol{e}_{h}\right) \\
& \left.+2 \sum_{h=1}^{k} \alpha_{h}\left(\boldsymbol{e}_{h} \circ \boldsymbol{e}_{h} \circ \boldsymbol{e}_{h}\right)\right) .
\end{aligned}
$$

Here $\boldsymbol{e}_{h}$ is standard basis vector of length $k$ with $h$ th coordinate being one. The third order moment tensor of $\boldsymbol{b}_{i j} \circ \boldsymbol{b}_{i s} \circ \boldsymbol{b}_{i t}$ can be derived as

$$
\begin{aligned}
\mathbb{E}\left(\boldsymbol{b}_{i j} \circ \boldsymbol{b}_{i s} \circ \boldsymbol{b}_{i t}\right)=\mathbb{E}\left(\boldsymbol{m}_{i j} \circ \boldsymbol{m}_{i s} \circ \boldsymbol{m}_{i t}\right) \times\left\{\boldsymbol{\Phi}_{j}, \boldsymbol{\Phi}_{s}, \boldsymbol{\Phi}_{t}\right\} \\
=\frac{1}{\alpha_{0}\left(\alpha_{0}+1\right)\left(\alpha_{0}+2\right)}\left(\left(\boldsymbol{\Phi}_{j} \boldsymbol{\alpha}\right) \circ\left(\boldsymbol{\Phi}_{s} \boldsymbol{\alpha}\right) \circ\left(\boldsymbol{\Phi}_{t} \boldsymbol{\alpha}\right)+\sum_{h=1}^{k} \alpha_{h}\left[\boldsymbol{\phi}_{j h} \circ \boldsymbol{\phi}_{s h} \circ\left(\boldsymbol{\Phi}_{t} \boldsymbol{\alpha}\right)\right]\right. \\
\quad+\sum_{h=1}^{k} \alpha_{h}\left[\boldsymbol{\phi}_{j h} \circ\left(\boldsymbol{\Phi}_{s} \boldsymbol{\alpha}\right) \circ \boldsymbol{\phi}_{t h}\right]+\sum_{h=1}^{k} \alpha_{h}\left[\left(\boldsymbol{\Phi}_{j} \boldsymbol{\alpha}\right) \circ \boldsymbol{\phi}_{s h} \circ \boldsymbol{\phi}_{t h}\right] \\
\left.\quad+2 \sum_{h=1}^{k} \alpha_{h} \boldsymbol{\phi}_{j h} \circ \boldsymbol{\phi}_{s h} \circ \boldsymbol{\phi}_{t h}\right) \\
=\frac{1}{\alpha_{0}\left(\alpha_{0}+1\right)\left(\alpha_{0}+2\right)}\left(\alpha_{0}^{3} \boldsymbol{\mu}_{j} \circ \boldsymbol{\mu}_{s} \circ \boldsymbol{\mu}_{t}\right. \\
\quad+\alpha_{0}^{2}\left(\alpha_{0}+1\right) \mathbb{E}\left(\boldsymbol{b}_{i j} \circ \boldsymbol{b}_{i s} \circ \boldsymbol{\mu}_{t}\right)-\alpha_{0}^{3} \boldsymbol{\mu}_{j} \circ \boldsymbol{\mu}_{s} \circ \boldsymbol{\mu}_{t} \\
\quad+\alpha_{0}^{2}\left(\alpha_{0}+1\right) \mathbb{E}\left(\boldsymbol{\mu}_{j} \circ \boldsymbol{b}_{i s} \circ \boldsymbol{b}_{i t}\right)-\alpha_{0}^{3} \boldsymbol{\mu}_{j} \circ \boldsymbol{\mu}_{s} \circ \boldsymbol{\mu}_{t} \\
+\alpha_{0}^{2}\left(\alpha_{0}+1\right) \mathbb{E}\left(\boldsymbol{b}_{i j} \circ \boldsymbol{\mu}_{s} \circ \boldsymbol{b}_{i t}\right)-\alpha_{0}^{3} \boldsymbol{\mu}_{j} \circ \boldsymbol{\mu}_{s} \circ \boldsymbol{\mu}_{t} \\
\left.\quad+2 \sum_{h=1}^{k} \alpha_{h} \boldsymbol{\phi}_{j h} \circ \boldsymbol{\phi}_{s h} \circ \boldsymbol{\phi}_{t h}\right) .
\end{aligned}
$$

The theorem follows Equations 17 and 18 . For non-categorical data, we let $\boldsymbol{b}_{i j} \equiv y_{i j}$ and $\boldsymbol{\phi}_{j h}$ is a scalar mean parameter for $y_{i j}$. Equations 17 and 18 still hold.

\section{B Proof of Theorem 2}

Proof. For notation simplicity we suppress $\boldsymbol{A}_{n}^{(\cdot)}$ in $Q_{n}^{(\cdot)}\left(\boldsymbol{\Phi} ; \boldsymbol{A}_{n}^{(\cdot)}\right)$ and $\boldsymbol{A}^{(\cdot)}$ in $Q_{0}^{(\cdot)}\left(\boldsymbol{\Phi} ; \boldsymbol{A}^{(\cdot)}\right)$. Lemma 1 implies $\lim _{n \rightarrow \infty} \operatorname{Pr}\left[\left|Q_{n}^{(\cdot)}\left(\widehat{\boldsymbol{\Phi}}^{(\cdot)}\right)-Q_{0}^{(\cdot)}\left(\widehat{\boldsymbol{\Phi}}^{(\cdot)}\right)\right|<\epsilon / 3\right]=1$ and $\lim _{n \rightarrow \infty} \operatorname{Pr}\left[\left|Q_{n}^{(\cdot)}\left(\boldsymbol{\Phi}_{0}\right)-Q_{0}^{(\cdot)}\left(\boldsymbol{\Phi}_{0}\right)\right|<\epsilon / 3\right]=1$ for $\epsilon>0$. This result also implies

$$
\begin{aligned}
& \lim _{n \rightarrow \infty} \operatorname{Pr}\left[Q_{0}^{(\cdot)}\left(\widehat{\boldsymbol{\Phi}}^{(\cdot)}\right)<Q_{n}^{(\cdot)}\left(\widehat{\boldsymbol{\Phi}}^{(\cdot)}\right)+\epsilon / 3\right]=1 . \\
& \lim _{n \rightarrow \infty} \operatorname{Pr}\left[Q_{n}^{(\cdot)}\left(\mathbf{\Phi}_{0}\right)<Q_{0}^{(\cdot)}\left(\mathbf{\Phi}_{0}\right)+\epsilon / 3\right]=1 .
\end{aligned}
$$

On the other hand, $\widehat{\boldsymbol{\Phi}}^{(\cdot)}$ minimizes $Q_{n}^{(\cdot)}(\boldsymbol{\Phi})$, therefore

$$
\lim _{n \rightarrow \infty} \operatorname{Pr}\left[Q_{n}^{(\cdot)}\left(\widehat{\boldsymbol{\Phi}}^{(\cdot)}\right)<Q_{n}^{(\cdot)}\left(\mathbf{\Phi}_{0}\right)+\epsilon / 3\right]=1 .
$$

Equations (19) and (21) imply

$$
\lim _{n \rightarrow \infty} \operatorname{Pr}\left[Q_{0}^{(\cdot)}\left(\widehat{\boldsymbol{\Phi}}^{(\cdot)}\right)<Q_{n}^{(\cdot)}\left(\boldsymbol{\Phi}_{0}\right)+2 \epsilon / 3\right]=1 .
$$

Together with Equation 20, we get

$$
\lim _{n \rightarrow \infty} \operatorname{Pr}\left[Q_{0}^{(\cdot)}\left(\widehat{\boldsymbol{\Phi}}^{(\cdot)}\right)<Q_{0}^{(\cdot)}\left(\mathbf{\Phi}_{0}\right)+\epsilon\right]=1 .
$$


Therefore

$$
\lim _{n \rightarrow \infty} \operatorname{Pr}\left[0 \leq Q_{0}^{(\cdot)}\left(\widehat{\boldsymbol{\Phi}}^{(\cdot)}\right)<\epsilon\right]=1
$$

follows with $Q_{0}^{(\cdot)}\left(\boldsymbol{\Phi}_{0}\right)=0$ and $Q_{0}^{(\cdot)}\left(\widehat{\boldsymbol{\Phi}}^{(\cdot)}\right) \geq 0$. Next, we choose a neighborhood $N$, which contains $\boldsymbol{\Phi}_{0}$ in $\boldsymbol{\Theta}$. Due to the compactness of $\boldsymbol{\Theta}$ the neighborhood $N^{C}$ is also compact. The continuousness of $Q_{0}^{(\cdot)}(\boldsymbol{\Phi})$ implies the existence of $\inf _{\boldsymbol{\Phi} \in N^{C}} Q_{0}^{(\cdot)}(\boldsymbol{\Phi})$ and it is positive. Let $\epsilon=\inf _{\boldsymbol{\Phi} \in N^{C}} Q_{0}^{(\cdot)}(\boldsymbol{\Phi})$, then we get

$$
\lim _{n \rightarrow \infty} \operatorname{Pr}\left[0 \leq Q_{0}^{(\cdot)}\left(\widehat{\boldsymbol{\Phi}}^{(\cdot)}\right)<\inf _{\boldsymbol{\Phi} \in N^{C}} Q_{0}^{(\cdot)}(\boldsymbol{\Phi})\right]=1 .
$$

Therefore $\lim _{n \rightarrow \infty} \operatorname{Pr}\left(\widehat{\boldsymbol{\Phi}}^{(\cdot)} \notin N^{C}\right)=1$, which suggests $\lim _{n \rightarrow \infty} \operatorname{Pr}\left(\widehat{\boldsymbol{\Phi}}^{(\cdot)} \in N\right)=1$. Shrinking the neighborhood size of $N$ we get

$$
\lim _{n \rightarrow \infty} \operatorname{Pr}\left(\widehat{\boldsymbol{\Phi}}^{(\cdot)}=\mathbf{\Phi}_{0}\right)=1
$$

\section{Proof of Theorem 3}

Proof. We approximate $\boldsymbol{f}_{n}^{(\cdot)}\left(\widehat{\boldsymbol{\Phi}}^{(\cdot)}\right)$ using first order Taylor expansion

$$
\boldsymbol{f}_{n}^{(\cdot)}\left(\widehat{\boldsymbol{\Phi}}^{(\cdot)}\right)=\boldsymbol{f}_{n}^{(\cdot)}\left(\boldsymbol{\Phi}_{0}\right)+\boldsymbol{G}_{n}^{(\cdot)}\left(\boldsymbol{\Phi}_{0}\right)\left[\operatorname{vec}\left(\widehat{\boldsymbol{\Phi}}^{(\cdot)}\right)-\operatorname{vec}\left(\boldsymbol{\Phi}_{0}\right)\right]+O\left\{\left[\operatorname{vec}\left(\widehat{\boldsymbol{\Phi}}^{(\cdot)}\right)-\operatorname{vec}\left(\boldsymbol{\Phi}_{0}\right)\right]^{2}\right\}
$$

Ignoring the high order term, we left multiply both sides by $\left[\boldsymbol{G}_{n}^{(\cdot)}\left(\widehat{\boldsymbol{\Phi}}^{(\cdot)}\right)\right]^{T} \boldsymbol{A}_{n}^{(\cdot)}$. Then we get

$$
\begin{aligned}
{\left[\boldsymbol{G}_{n}^{(\cdot)}\left(\widehat{\boldsymbol{\Phi}}^{(\cdot)}\right)\right]^{T} \boldsymbol{A}_{n}^{(\cdot)} \boldsymbol{f}_{n}^{(\cdot)}\left(\widehat{\boldsymbol{\Phi}}^{(\cdot)}\right) \approx\left[\boldsymbol{G}_{n}^{(\cdot)}\left(\widehat{\boldsymbol{\Phi}}^{(\cdot)}\right)\right]^{T} \boldsymbol{A}_{n}^{(\cdot)} \boldsymbol{f}_{n}^{(\cdot)}\left(\boldsymbol{\Phi}_{0}\right) } \\
+\left[\boldsymbol{G}_{n}^{(\cdot)}\left(\widehat{\boldsymbol{\Phi}}^{(\cdot)}\right)\right]^{T} \boldsymbol{A}_{n}^{(\cdot)} \boldsymbol{G}_{n}^{(\cdot)}\left(\boldsymbol{\Phi}_{0}\right)\left[\operatorname{vec}\left(\widehat{\boldsymbol{\Phi}}^{(\cdot)}\right)-\operatorname{vec}\left(\boldsymbol{\Phi}_{0}\right)\right]
\end{aligned}
$$

The fact that estimator $\widehat{\boldsymbol{\Phi}}^{(\cdot)}$ minimizes $Q_{n}^{(\cdot)}\left(\boldsymbol{\Phi}, \boldsymbol{A}_{n}^{(\cdot)}\right)$ implies the left hand side equals to zero. Therefore we get

$$
n^{1 / 2}\left[\operatorname{vec}\left(\widehat{\boldsymbol{\Phi}}^{(\cdot)}\right)-\operatorname{vec}\left(\boldsymbol{\Phi}_{0}\right)\right] \approx-\left\{\left[\boldsymbol{G}_{n}^{(\cdot)}\left(\widehat{\boldsymbol{\Phi}}^{(\cdot)}\right)\right]^{T} \boldsymbol{A}_{n}^{(\cdot)} \boldsymbol{G}_{n}^{(\cdot)}\left(\boldsymbol{\Phi}_{0}\right)\right\}^{-1}\left[\boldsymbol{G}_{n}^{(\cdot)}\left(\widehat{\boldsymbol{\Phi}}^{(\cdot)}\right)\right]^{T} \boldsymbol{A}_{n}^{(\cdot)} n^{1 / 2} \boldsymbol{f}_{n}^{(\cdot)}\left(\boldsymbol{\Phi}_{0}\right)
$$

The theorem follows with $n^{1 / 2} \boldsymbol{f}_{n}^{(\cdot)}\left(\boldsymbol{\Phi}_{0}\right) \stackrel{p}{\rightarrow} \mathcal{N}\left(\mathbf{0}, \boldsymbol{S}^{(\cdot)}\right)$ and Assumptions 1 and 2

\section{Derivatives of moment functions}

\section{D.1 Second moment matrix}

The second moment matrix $\boldsymbol{F}_{j t}^{(2)}\left(\boldsymbol{y}_{i}, \boldsymbol{\Phi}\right)$ may be written as $\boldsymbol{b}_{i j} \circ \boldsymbol{b}_{i t}-\boldsymbol{\Phi}_{j} \mathbb{E}\left(\boldsymbol{x}_{i} \circ \boldsymbol{x}_{i}\right) \boldsymbol{\Phi}_{t}^{T}$. The derivatives of $\boldsymbol{F}_{j t}^{(2)}\left(\boldsymbol{y}_{i}, \boldsymbol{\Phi}\right)$ with respect to $\boldsymbol{\Phi}_{j}$ and $\boldsymbol{\Phi}_{t}$ can be written as

$$
\begin{aligned}
\frac{\partial \operatorname{vec}\left[\boldsymbol{F}_{j t}^{(2)}\left(\boldsymbol{y}_{i}, \boldsymbol{\Phi}\right)\right]}{\partial \operatorname{vec}\left(\boldsymbol{\Phi}_{j}\right)} & =-\frac{\partial\left\{\left[\boldsymbol{\Phi}_{t} \mathbb{E}\left(\boldsymbol{x}_{i} \circ \boldsymbol{x}_{i}\right)\right] \otimes \boldsymbol{I}_{d_{j}}\right\} \operatorname{vec}\left(\boldsymbol{\Phi}_{j}\right)}{\partial \operatorname{vec}\left(\boldsymbol{\Phi}_{j}\right)} \\
& =-\left[\boldsymbol{\Phi}_{t} \mathbb{E}\left(\boldsymbol{x}_{i} \circ \boldsymbol{x}_{i}\right)\right] \otimes \boldsymbol{I}_{d_{j}}, \\
\frac{\partial \operatorname{vec}\left[\boldsymbol{F}_{j t}^{(2)}\left(\boldsymbol{y}_{i}, \boldsymbol{\Phi}\right)\right]}{\partial \operatorname{vec}\left(\boldsymbol{\Phi}_{t}\right)} & =-\boldsymbol{T} \frac{\partial \operatorname{vec}\left[\boldsymbol{\Phi}_{t} \mathbb{E}\left(\boldsymbol{x}_{i} \circ \boldsymbol{x}_{i}\right) \boldsymbol{\Phi}_{j}^{T}\right]}{\partial \operatorname{vec}\left(\boldsymbol{\Phi}_{t}\right)} \\
& =-\boldsymbol{T} \frac{\partial\left\{\left[\boldsymbol{\Phi}_{j} \mathbb{E}\left(\boldsymbol{x}_{i} \circ \boldsymbol{x}_{i}\right)\right] \otimes \boldsymbol{I}_{d_{t}} \operatorname{vec}\left(\boldsymbol{\Phi}_{t}\right)\right\}}{\partial \operatorname{vec}\left(\boldsymbol{\Phi}_{t}\right)} \\
& =-\boldsymbol{T}\left\{\left[\boldsymbol{\Phi}_{j} \mathbb{E}\left(\boldsymbol{x}_{i} \circ \boldsymbol{x}_{i}\right)\right] \otimes \boldsymbol{I}_{d_{t}}\right\}
\end{aligned}
$$


where $\otimes$ indicates a Kronecker product and $\boldsymbol{T}$ is a $d_{t} k \times d_{t} k 0 / 1$ matrix that satisfies

$$
\operatorname{vec}\left[\boldsymbol{\Phi}_{j} \mathbb{E}\left(\boldsymbol{x}_{i} \circ \boldsymbol{x}_{i}\right) \boldsymbol{\Phi}_{t}^{T}\right]=\boldsymbol{T}_{\operatorname{vec}}\left[\boldsymbol{\Phi}_{t} \mathbb{E}\left(\boldsymbol{x}_{i} \circ \boldsymbol{x}_{i}\right) \boldsymbol{\Phi}_{j}^{T}\right]
$$

Therefore $\mathbb{E}\left[\partial \boldsymbol{f}^{(2)}\left(\boldsymbol{y}_{i}, \boldsymbol{\Phi}\right) / \partial \boldsymbol{\Phi}\right]$ is a block matrix with block of $-\boldsymbol{\Phi}_{t}^{T} \mathbb{E}\left(\boldsymbol{x}_{i} \circ \boldsymbol{x}_{i}\right) \otimes \boldsymbol{I}_{d_{j}}$ on columns corresponding to $\operatorname{vec}\left(\boldsymbol{\Phi}_{t}\right)$ and rows corresponding to $\operatorname{vec}\left[\boldsymbol{F}_{j t}^{(2)}\left(\boldsymbol{y}_{i}, \boldsymbol{\Phi}\right)\right]$.

\section{D.2 Third moment tensor}

We next consider the third moment tensor. Write $\boldsymbol{F}_{j s t}^{(3)}\left(\boldsymbol{y}_{i}, \boldsymbol{\Phi}\right)$ as $\boldsymbol{b}_{i j} \circ \boldsymbol{b}_{i j} \circ \boldsymbol{b}_{i j}-\mathbb{E}\left(\boldsymbol{x}_{i} \circ \boldsymbol{x}_{i} \circ \boldsymbol{x}_{i}\right) \times_{1} \boldsymbol{\Phi}_{j} \times_{2}$ $\boldsymbol{\Phi}_{s} \times_{3} \boldsymbol{\Phi}_{t}$. Then only the second term involves $\boldsymbol{\Phi}$.

The derivatives of $\boldsymbol{F}_{j s t}^{(3)}\left(\boldsymbol{y}_{i}, \boldsymbol{\Phi}\right)$ with respect to $\boldsymbol{\Phi}_{j}$ can be written as

$$
\begin{aligned}
\frac{\partial \operatorname{vec}\left[\boldsymbol{F}_{j s t}^{(3)}\left(\boldsymbol{y}_{i}, \boldsymbol{\Phi}\right)\right]}{\partial \operatorname{vec}\left(\boldsymbol{\Phi}_{j}\right)} & =-\frac{\partial \operatorname{vec}\left\{\left[\mathbb{E}\left(\boldsymbol{x}_{i} \circ \boldsymbol{x}_{i} \circ \boldsymbol{x}_{i}\right) \times_{1} \boldsymbol{\Phi}_{j} \times_{2} \boldsymbol{\Phi}_{s} \times_{3} \boldsymbol{\Phi}_{t}\right]_{(1)}\right\}}{\partial \operatorname{vec}\left(\boldsymbol{\Phi}_{j}\right)} \\
& =-\frac{\partial \operatorname{vec}\left\{\left[\boldsymbol{\Phi}_{j} \mathbb{E}\left(\boldsymbol{x}_{i} \circ \boldsymbol{x}_{i} \circ \boldsymbol{x}_{i}\right)_{(1)}\left(\boldsymbol{\Phi}_{t} \otimes \boldsymbol{\Phi}_{s}\right)^{T}\right\}\right.}{\partial \operatorname{vec}\left(\boldsymbol{\Phi}_{j}\right)} \\
& =-\left(\boldsymbol{\Phi}_{t} \otimes \boldsymbol{\Phi}_{s}\right) \operatorname{vec}\left[\mathbb{E}\left(\boldsymbol{x}_{i} \circ \boldsymbol{x}_{i} \circ \boldsymbol{x}_{i}\right)_{(1)}\right]^{T} \otimes \boldsymbol{I}_{d_{j}},
\end{aligned}
$$

where subscript (1) indicates model-1 unfolding of a three way tensor.

The derivatives of $\boldsymbol{F}_{j s t}^{(3)}\left(\boldsymbol{y}_{i}, \boldsymbol{\Phi}\right)$ with respect to $\boldsymbol{\Phi}_{s}$ and $\boldsymbol{\Phi}_{t}$ can be calculated accordingly by introducing 0/1 transformation matrices $\boldsymbol{T}_{(2)(1)}$ and $\boldsymbol{T}_{(3)(1)}$ both with size $d_{j} d_{s} d_{t} \times d_{j} d_{s} d_{t}$ that satisfy

$$
\begin{aligned}
\operatorname{vec}\left\{\left[\boldsymbol{F}_{j s t}^{(3)}\left(\boldsymbol{y}_{i}, \boldsymbol{\Phi}\right)\right]_{(1)}\right\} & =\boldsymbol{T}_{(2)(1)} \operatorname{vec}\left\{\left[\boldsymbol{F}_{j s t}^{(3)}\left(\boldsymbol{y}_{i}, \boldsymbol{\Phi}\right)\right]_{(2)}\right\} \\
& =\boldsymbol{T}_{(3)(1)} \operatorname{vec}\left\{\left[\boldsymbol{F}_{j s t}^{(3)}\left(\boldsymbol{y}_{i}, \boldsymbol{\Phi}\right)\right]_{(3)}\right\} .
\end{aligned}
$$

Then

$$
\begin{aligned}
\frac{\partial \operatorname{vec}\left[\boldsymbol{F}_{j s t}^{(3)}\left(\boldsymbol{y}_{i}, \boldsymbol{\Phi}\right)\right]}{\partial \operatorname{vec}\left(\boldsymbol{\Phi}_{s}\right)} & =-\boldsymbol{T}_{(2)(1)} \frac{\partial \operatorname{vec}\left\{\left[\mathbb{E}\left(\boldsymbol{x}_{i} \circ \boldsymbol{x}_{i} \circ \boldsymbol{x}_{i}\right) \times_{1} \boldsymbol{\Phi}_{j} \times_{2} \boldsymbol{\Phi}_{s} \times_{3} \boldsymbol{\Phi}_{t}\right]_{(2)}\right\}}{\partial \operatorname{vec}\left(\boldsymbol{\Phi}_{s}\right)} \\
& =-\boldsymbol{T}_{(2)(1)} \frac{\partial \operatorname{vec}\left\{\left[\boldsymbol{\Phi}_{s} \mathbb{E}\left(\boldsymbol{x}_{i} \circ \boldsymbol{x}_{i} \circ \boldsymbol{x}_{i}\right)_{(2)}\left(\boldsymbol{\Phi}_{t} \otimes \boldsymbol{\Phi}_{j}\right)^{T}\right\}\right.}{\partial \operatorname{vec}\left(\boldsymbol{\Phi}_{s}\right)} \\
& =-\boldsymbol{T}_{(2)(1)}\left\{\left(\boldsymbol{\Phi}_{t} \otimes \boldsymbol{\Phi}_{j}\right)\left[\mathbb{E}\left(\boldsymbol{x}_{i} \circ \boldsymbol{x}_{i} \circ \boldsymbol{x}_{i}\right)_{(2)}\right]^{T} \otimes \boldsymbol{I}_{d_{s}}\right\}, \\
\frac{\partial \operatorname{vec}\left[\boldsymbol{F}_{j s t}^{(3)}\left(\boldsymbol{y}_{i}, \boldsymbol{\Phi}\right)\right]}{\partial \operatorname{vec}\left(\boldsymbol{\Phi}_{t}\right)} & =-\boldsymbol{T}_{(3)(1)} \frac{\partial \operatorname{vec}\left\{\left[\mathbb{E}\left(\boldsymbol{x}_{i} \circ \boldsymbol{x}_{i} \circ \boldsymbol{x}_{i}\right) \times_{1} \boldsymbol{\Phi}_{j} \times_{2} \boldsymbol{\Phi}_{s} \times_{3} \boldsymbol{\Phi}_{t}\right]_{(3)}\right\}}{\partial \operatorname{vec}\left(\boldsymbol{\Phi}_{t}\right)} \\
& =-\boldsymbol{T}_{(3)(1)} \frac{\partial \operatorname{vec}\left\{\left[\boldsymbol{\Phi}_{t} \mathbb{E}\left(\boldsymbol{x}_{i} \circ \boldsymbol{x}_{i} \circ \boldsymbol{x}_{i}\right)_{(3)}\left(\boldsymbol{\Phi}_{s} \otimes \boldsymbol{\Phi}_{j}\right)^{T}\right\}\right.}{\partial \operatorname{vec}\left(\boldsymbol{\Phi}_{t}\right)} \\
& =-\boldsymbol{T}_{(3)(1)}\left\{\left(\boldsymbol{\Phi}_{s} \otimes \boldsymbol{\Phi}_{j}\right)\left[\mathbb{E}\left(\boldsymbol{x}_{i} \circ \boldsymbol{x}_{i} \circ \boldsymbol{x}_{i}\right)_{(3)}\right]^{T} \otimes \boldsymbol{I}_{d_{t}}\right\} .
\end{aligned}
$$

The conditions 1), 3) and 4) in Assumption 2 in main text follow after we calculating the derivatives of moment functions.

\section{E Derivation of Newton-Raphson update}

We denote

$$
\begin{aligned}
\boldsymbol{E}_{n, j t}^{(2)} & =\boldsymbol{F}_{n, j t}^{(2)}(\boldsymbol{\Phi})+\boldsymbol{\Phi}_{j} \boldsymbol{\Lambda}^{(2)} \boldsymbol{\Phi}_{t}^{T} \\
\boldsymbol{E}_{n, j s t}^{(3)} & =\boldsymbol{F}_{n, j s t}^{(3)}(\boldsymbol{\Phi})+\boldsymbol{\Lambda}^{(3)} \times_{1} \boldsymbol{\Phi}_{j} \times_{2} \boldsymbol{\Phi}_{s} \times_{3} \boldsymbol{\Phi}_{t} .
\end{aligned}
$$


With identity matrix, then the two objective functions can be written as

$$
\begin{aligned}
Q_{n}^{(2)}(\boldsymbol{\Phi})= & \sum_{j=1}^{p-1} \sum_{t=j+1}^{p}\left\|\boldsymbol{E}_{n, j t}^{(2)}-\boldsymbol{\Phi}_{j} \boldsymbol{\Lambda}^{(2)} \boldsymbol{\Phi}_{t}^{T}\right\|_{F}^{2}, \\
Q_{n}^{(3)}(\boldsymbol{\Phi})= & \sum_{j=1}^{p-1} \sum_{t=j+1}^{p}\left\|\boldsymbol{E}_{n, j t}^{(2)}-\boldsymbol{\Phi}_{j} \boldsymbol{\Lambda}^{(2)} \boldsymbol{\Phi}_{t}^{T}\right\|_{F}^{2} \\
& +\sum_{j=1}^{p-2} \sum_{s=j+1}^{p-1} \sum_{t=s+1}^{p}\left\|\boldsymbol{E}_{n, j s t}^{(3)}-\boldsymbol{\Lambda}^{(3)} \times_{1} \boldsymbol{\Phi}_{j} \times_{2} \boldsymbol{\Phi}_{s} \times_{3} \boldsymbol{\Phi}_{t}\right\|_{F}^{2} .
\end{aligned}
$$

Here we suppress the weight matrix in the objective functions. We first consider $Q_{n}^{(2)}(\boldsymbol{\Phi})$. The terms involve $\phi_{j h}$ are

$$
\sum_{t=1, t \neq j}^{p}\left[-2 \lambda_{h}^{(2)}\left(\overline{\boldsymbol{E}}_{n, j t}^{(2)} \boldsymbol{\phi}_{t h}\right)^{T} \boldsymbol{\phi}_{j h}+\left(\lambda_{h}^{(2)}\right)^{2}\left(\boldsymbol{\phi}_{t h}^{T} \boldsymbol{\phi}_{t h}\right) \boldsymbol{\phi}_{j h}^{T} \boldsymbol{\phi}_{j h}\right]
$$

where $\overline{\boldsymbol{E}}_{n, j t}^{(2)}=\boldsymbol{E}_{n, j t}^{(2)}-\sum_{h^{\prime} \neq h} \lambda_{h^{\prime}}^{(2)} \boldsymbol{\phi}_{j h^{\prime}} \circ \boldsymbol{\phi}_{t h^{\prime}}$ and $\lambda_{h}^{(2)}$ is the $h$ th diagonal element of $\boldsymbol{\Lambda}^{(2)}$. Here we use the fact that $\left\|\boldsymbol{E}_{n, j t}^{(2)}-\boldsymbol{\Phi}_{j} \Lambda^{(2)} \boldsymbol{\Phi}_{t}^{T}\right\|_{F}^{2}=\left\|\boldsymbol{E}_{n, t j}^{(2)}-\boldsymbol{\Phi}_{t} \Lambda^{(2)} \boldsymbol{\Phi}_{j}^{T}\right\|_{F}^{2}$. By letting

$$
\begin{aligned}
& \boldsymbol{\xi}^{(2)}=-2 \lambda_{h}^{(2)} \sum_{t=1, t \neq j}^{p}\left(\overline{\boldsymbol{E}}_{n, j t}^{(2)} \boldsymbol{\phi}_{t h}\right), \\
& \gamma^{(2)}=\left(\lambda_{h}^{(2)}\right)^{2} \sum_{t=1, t \neq j}^{p} \boldsymbol{\phi}_{t h}^{T} \boldsymbol{\phi}_{t h},
\end{aligned}
$$

the gradient $\nabla Q_{n}^{(2)}\left(\phi_{j h}\right)$ and Hessian $\nabla^{2} Q_{n}^{(2)}\left(\phi_{j h}\right)$ can be written as

$$
\begin{aligned}
\nabla Q_{n}^{(2)}\left(\boldsymbol{\phi}_{j h}\right) & =\boldsymbol{\xi}^{(2)}+2 \gamma^{(2)} \boldsymbol{\phi}_{j h} \\
\nabla^{2} Q_{n}^{(2)}\left(\boldsymbol{\phi}_{j h}\right) & =2 \gamma^{(2)} \boldsymbol{I}
\end{aligned}
$$

The update rule in 11 can be derived accordingly.

Then we consider $Q_{n}^{(3)}(\boldsymbol{\Phi})$. The terms involve $\boldsymbol{\phi}_{j h}$ are

$$
\begin{aligned}
& \sum_{t=1, t \neq j}^{p}\left[-2 \lambda_{h}^{(2)}\left(\overline{\boldsymbol{E}}_{n, j t}^{(2)} \boldsymbol{\phi}_{t h}\right)^{T} \boldsymbol{\phi}_{j h}+\left(\lambda_{h}^{(2)}\right)^{2}\left(\boldsymbol{\phi}_{t h}^{T} \boldsymbol{\phi}_{t h}\right) \boldsymbol{\phi}_{j h}^{T} \boldsymbol{\phi}_{j h}\right] \\
& \quad+\sum_{s=1, s \neq j}^{p} \sum_{t=1, t \neq s, t \neq j}^{p}\left[-2\left\langle\overline{\boldsymbol{E}}_{n, j s t}^{(3)}, \lambda_{h}^{(3)} \boldsymbol{\phi}_{j h} \circ \boldsymbol{\phi}_{s h} \circ \boldsymbol{\phi}_{t h}\right\rangle+\left\|\lambda_{h}^{(3)} \boldsymbol{\phi}_{j h} \circ \boldsymbol{\phi}_{s h} \circ \boldsymbol{\phi}_{t h}\right\|_{F}^{2}\right],
\end{aligned}
$$

where $\overline{\boldsymbol{E}}_{n, j t}^{(2)}=\boldsymbol{E}_{n, j t}^{(2)}-\sum_{h^{\prime} \neq h} \lambda_{h^{\prime}}^{(2)} \boldsymbol{\phi}_{j h^{\prime}} \circ \boldsymbol{\phi}_{t h^{\prime}}$ and $\overline{\boldsymbol{E}}_{n, j s t}^{(3)}=\boldsymbol{E}_{n, j s t}^{(3)}-\sum_{h^{\prime} \neq h} \lambda_{h^{\prime}}^{(3)} \boldsymbol{\phi}_{j h^{\prime}} \circ \boldsymbol{\phi}_{s h^{\prime}} \circ \boldsymbol{\phi}_{t h^{\prime}}$. Again we use the symmetric property of $\left\|\boldsymbol{E}_{n, j t}^{(2)}-\boldsymbol{\Phi}_{j} \boldsymbol{\Lambda}^{(2)} \boldsymbol{\Phi}_{t}^{T}\right\|_{F}^{2}$ and $\left\|\boldsymbol{E}_{n, j s t}^{(3)}-\boldsymbol{\Lambda}^{(3)} \times_{1} \boldsymbol{\Phi}_{j} \times_{2} \boldsymbol{\Phi}_{s} \times_{3} \boldsymbol{\Phi}_{t}\right\|_{F}^{2}$. By organizing the terms, we get

$$
\begin{aligned}
\sum_{t=1, t \neq j}^{p}\left[-2 \lambda_{h}^{(2)}\left(\overline{\boldsymbol{E}}_{n, j t}^{(2)} \boldsymbol{\phi}_{t h}\right)^{T} \boldsymbol{\phi}_{j h}+\left(\lambda_{h}^{(2)}\right)^{2}\left(\boldsymbol{\phi}_{t h}^{T} \boldsymbol{\phi}_{t h}\right) \boldsymbol{\phi}_{j h}^{T} \boldsymbol{\phi}_{j h}\right] & \\
& +\sum_{s=1, s \neq j}^{p} \sum_{t=1, t \neq s, t \neq j}^{p}\left[-2 \lambda_{h}^{(3)}\left(\overline{\boldsymbol{E}}_{n, j s t}^{(3)} \times_{2} \boldsymbol{\phi}_{s h} \times{ }_{3} \boldsymbol{\phi}_{t h}\right)^{T} \boldsymbol{\phi}_{j h}+\left(\lambda_{h}^{(3)}\right)^{2}\left(\boldsymbol{\phi}_{s h}^{T} \boldsymbol{\phi}_{s h}\right)\left(\boldsymbol{\phi}_{t h}^{T} \boldsymbol{\phi}_{t h}\right)\left(\boldsymbol{\phi}_{j h}^{T} \boldsymbol{\phi}_{j h}\right)\right] .
\end{aligned}
$$

By letting

$$
\boldsymbol{\xi}^{(3)}=-2 \lambda_{h}^{(2)} \sum_{t=1, t \neq j}^{p}\left(\overline{\boldsymbol{E}}_{n, j t}^{(2)} \boldsymbol{\phi}_{t h}\right)-2 \lambda_{h}^{(3)} \sum_{s=1, s \neq j}^{p}\left[\sum_{t=1, t \neq s, t \neq j}^{p}\left(\overline{\boldsymbol{E}}_{n, j s t}^{(3)} \times_{2} \boldsymbol{\phi}_{s h} \times{ }_{3} \boldsymbol{\phi}_{t h}\right)\right]
$$




$$
\gamma^{(3)}=\left(\lambda_{h}^{(2)}\right)^{2} \sum_{t=1, t \neq j}^{p} \phi_{t h}^{T} \phi_{t h}+\left(\lambda_{h}^{(3)}\right)^{2} \sum_{s=1, s \neq j}^{p}\left[\sum_{t=1, t \neq s, t \neq j}^{p}\left(\phi_{s h}^{T} \phi_{s h}\right)\left(\phi_{t h}^{T} \phi_{t h}\right)\right],
$$

the gradient $\nabla Q_{n}^{(3)}\left(\phi_{j h}\right)$ and Hessian $\nabla^{2} Q_{n}^{(3)}\left(\phi_{j h}\right)$ can be written as

$$
\begin{aligned}
\nabla Q_{n}^{(3)}\left(\boldsymbol{\phi}_{j h}\right) & =\boldsymbol{\xi}^{(3)}+2 \gamma^{(3)} \boldsymbol{\phi}_{j h} \\
\nabla^{2} Q_{n}^{(3)}\left(\boldsymbol{\phi}_{j h}\right) & =2 \gamma^{(3)} \boldsymbol{I}
\end{aligned}
$$

The update rule in 12 follows directly.

\section{References}

Anandkumar, A., Ge, R., Hsu, D., and Kakade, S. M. (2014a). A tensor approach to learning mixed membership community models. The Journal of Machine Learning Research, 15:2239-2312.

Anandkumar, A., Ge, R., Hsu, D., Kakade, S. M., and Telgarsky, M. (2014b). Tensor decompositions for learning latent variable models. The Journal of Machine Learning Research, 15:2773-2832.

Anandkumar, A., Hsu, D., and Kakade, S. M. (2012a). A method of moments for mixture models and hidden markov models. JMLR WECP 23: COLT.

Anandkumar, A., Liu, Y., Hsu, D. J., Foster, D. P., and Kakade, S. M. (2012b). A spectral algorithm for latent Dirichlet allocation. In Advances in Neural Information Processing Systems 25, pages 917-925.

Anderson, J. C. and Gerbing, D. W. (1988). Structural equation modeling in practice: A review and recommended two-step approach. Psychological bulletin, 103(3):411.

Arora, S., Ge, R., and Moitra, A. (2012). Learning topic models - going beyond SVD. In Fifty-Third IEEE Annual Symposium on Foundations of Computer Science, pages 1-10.

Bentler, P. M. (1983). Some contributions to efficient statistics in structural models: Specification and estimation of moment structures. Psychometrika, 48(4):493-517.

Bhattacharya, A. and Dunson, D. B. (2012). Simplex factor models for multivariate unordered categorical data. Journal of the American Statistical Association, 107(497):362-377.

Blei, D. M., Ng, A. Y., and Jordan, M. I. (2003). Latent Dirichlet allocation. The Journal of Machine Learning Research, 3:993-1022.

Bollen, K. A., Kolenikov, S., and Bauldry, S. (2014). Model-implied instrumental variable-generalized method of moments (MIIV-GMM) estimators for latent variable models. Psychometrika, 79(1):20-50.

Chang, J. (2012). lda: Collapsed Gibbs sampling methods for topic models.

Colombo, N. and Vlassis, N. (2015). FastMotif: Spectral sequence motif discovery. Bioinformatics, to appear.

Dunson, D. B. (2003). Dynamic latent trait models for multidimensional longitudinal data. Journal of the American Statistical Association, 98(463):555-563. 
Dunson, D. B. and Xing, C. (2009). Nonparametric Bayes modeling of multivariate categorical data. Journal of the American Statistical Association, 104(487):1042-1051.

Gallant, A. R., Giacomini, R., and Ragusa, G. (2013). Generalized Method of Moments with Latent Variables. Centre for Economic Policy Research.

Hall, A. R. (2005). Generalized Method of Moments. Oxford University Press.

Hansen, L. P. (1982). Large sample properties of generalized method of moments estimators. Econometrica: Journal of the Econometric Society, 50(4):1029.

Harley, C. B. and Reynolds, R. P. (1987). Analysis of E. coli promoter sequences. Nucleic Acids Research, $15(5): 2343-2361$.

Hoff, P. D. (2007). Extending the rank likelihood for semiparametric copula estimation. The Annals of Applied Statistics, 1(1):265-283.

Hsu, D. and Kakade, S. M. (2013). Learning mixtures of spherical Gaussians: Moment methods and spectral decompositions. In Proceedings of the 4 th conference on Innovations in Theoretical Computer Science, pages 11-20. ACM.

Jöreskog, K. G. and Sörbom, D. (1987). New developments in LISREL. Paper presented at the National Symposium on Methodological Issues in Causal Modeling, University of Alabama, Tuscaloosa.

Kiers, H. A. (2000). Towards a standardized notation and terminology in multiway analysis. Journal of chemometrics, 14(3):105-122.

Lichman, M. (2013). UCI machine learning repository.

Moustaki, I. and Knott, M. (2000). Generalized latent trait models. Psychometrika, 65(3):391-411.

Murray, J. S., Dunson, D. B., Carin, L., and Lucas, J. E. (2013). Bayesian Gaussian copula factor models for mixed data. Journal of the American Statistical Association, 108(502):656-665.

Muthén, B. (1984). A general structural equation model with dichotomous, ordered categorical, and continuous latent variable indicators. Psychometrika, 49(1):115-132.

Pritchard, J. K., Stephens, M., and Donnelly, P. (2000a). Inference of population structure using multilocus genotype data. Genetics, 155(2):945-959.

Pritchard, J. K., Stephens, M., Rosenberg, N. A., and Donnelly, P. (2000b). Association mapping in structured populations. The American Journal of Human Genetics, 67(1):170-181.

Quinn, K. M. (2004). Bayesian factor analysis for mixed ordinal and continuous responses. Political Analysis, 12(4):338-353.

Sammel, M. D., Ryan, L. M., and Legler, J. M. (1997). Latent variable models for mixed discrete and continuous outcomes. Journal of the Royal Statistical Society. Series B, 59(3):667-678.

Tung, H. F. and Smola, A. J. (2014). Spectral methods for Indian buffet process inference. In Advances in Neural Information Processing Systems 27, pages 1484-1492. 